\title{
Electrically driven convection in a thin annular film undergoing circular Couette flow
}

\author{
Zahir A. Daya, V.B. Deyirmenjian, and Stephen W. Morris \\ Department of Physics, University of Toronto, Toronto, Ontario, Canada, M5S 1A7
}

\begin{abstract}
We investigate the linear stability of a thin, suspended, annular film of conducting fluid with a voltage difference applied between its inner and outer edges. For a sufficiently large voltage, such a film is unstable to radially-driven electroconvection due to charges which develop on its free surfaces. The film can also be subjected to a Couette shear by rotating its inner edge. This combination is experimentally realized using films of smectic A liquid crystals. In the absence of shear, the convective flow consists of a stationary, azimuthally one-dimensional pattern of symmetric, counter-rotating vortex pairs. When Couette flow is applied, an azimuthally traveling pattern results. When viewed in a co-rotating frame, the traveling pattern consists of pairs of asymmetric vortices. We calculate the neutral stability boundary for arbitrary radius ratio $\alpha$ and Reynolds number $\mathcal{R} e$ of the shear flow, and obtain the critical control parameter $\mathcal{R}_{c}(\alpha, \mathcal{R} e)$ and the critical azimuthal mode number $m_{c}(\alpha, \mathcal{R} e)$. The Couette flow suppresses the onset of electroconvection, so that $\mathcal{R}_{c}(\alpha, \mathcal{R} e)>\mathcal{R}_{c}(\alpha, 0)$. The calculated suppression is compared with experiments performed at $\alpha=0.56$ and $0 \leq \mathcal{R} e \leq 0.22$.
\end{abstract}

Physics of Fluids, 11, 3613 (1999). See also http://mobydick.physics.utoronto.ca.

\section{INTRODUCTION}

We examine the effect of a circular Couette shear on radially driven convection in a two-dimensional annular fluid. Surprizingly, this remarkably ideal situation is experimentally realizeable in the electroconvection of thin, freely suspended liquid crystal films. [1] This system, which can be accurately described by electrohydrodynamic theory [2,3], presents a unique opportunity to quantitatively study convection with a novel combination of forcing and shear. In this paper, our treatment is both theoretical and experimental; we present a complete linear stability analysis and use it to make the first quantitative comparisons with experiment. [1] This rather simple instablity has a rich phenomenolgy in its nonlinear behaviour, and is a promising new testing-ground for theories of bifurcations in nonlinear patterns. [4] Since it involves radial driving forces, this system may also be interesting as an experimental analog for some geophysical instabilities. [5, 6]

The linear instability and subsequent nonlinear evolution of flows depend strongly on the symmetry and structure of the unstable base flow. One way of systematically studying this dependence is to superpose simple flows or rotations on well-understood instabilities. For example, the standard case of buoyancy-driven Rayleigh-Bénard convection (RBC) has been studied in the presence of rotation [7,8, 9, 10] and shearing due to an open throughflow [11, 12], as well as in the geophysically interesting cases of radial gravitation with rotation [5]6]. The phenomenology of two-dimensional (2D) electroconvection in a rectangular geometry was the subject of our previous experimental [13, 14, 15, 16, 17] and theoretical [2,3] work. These studies made precise the degree of analogy with RBC; the electrical nature of the forcing introduces some crucial differences in detail, even at the level of linear stability. Here we superpose this basic instability on a shear in the form of a circular Couette flow. The annular geometry and the Couette shear naturally bring to mind another standard instability, that of Couette flow between concentric cylinders, which is unstable to three-dimensional (3D) Taylor Vortex flow (TVF). [7] TVF has also been examined for the cases of an imposed axial through-flow 18 and superposed on radial electrical driving [19]. Although our analysis involves a similar cylindrical geometry, the crucial difference in our case is that the flow is truly 2D, which prevents the 3D Taylor instability. 20,21] We will show, in fact, that the Couette shear is always stabilizing in our system.

In summary, the unique fluid-mechanical features of our system are that it achieves the superposition of a circular Couette shear with a radially (transverse to the shear) driven convective instability in an entirely 2D flow. This combination cannot be accurately realized in any other experimentally accessible system. We will show that this combination leads to some interesting new phenomena.

Electroconvection results when an applied electric field acts on space charges within a fluid, resulting in body forces which drive flows. Electroconvection in 3D isotropic dielectric fluids has been the subject of many previous studies. 222] In general, the agreement between experiment and theory has been modest, owing to difficulties in modeling the generation of charge at the electrodes.

Another often-studied form of electroconvection occurs 


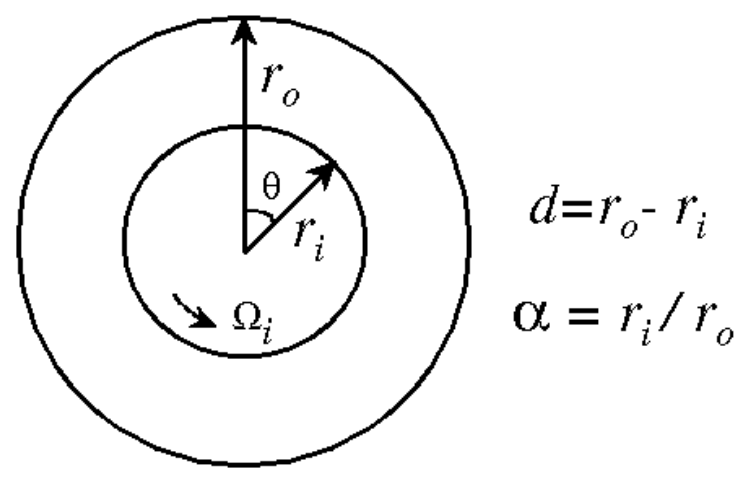

FIG. 1. Schematic of an annular film and cylindrical coordinate system. The film has a thickness $s \ll d$ in the $z$ direction (out of the page), and is treated as a two-dimensional sheet.

in certain anisotropic 3D fluids, i.e. liquid crystals, in which bulk charge separation occurs due to a mechanism which is unique to anisotropic fluid conductors. 223] Since this mechanism does not involve electrode effects, significant agreement between theory and experiment has been achieved despite the complexity of the anisotropic fluid.

In thin, suspended fluid films, another mechanism of charge separation can take place, leading to $2 \mathrm{D}$ electroconvection. If a voltage is applied across a conducting fluid film, the free surfaces acquire a potentially unstable distribution of surface charge. [2,3] This charge must be present simply to satisfy the standard matching conditions on electric fields across an interface. We do not consider charge generation by electrode injection or other bulk effects. A non-zero threshold voltage for the onset of electroconvection results from the competition between the electrical force on the surface charge configuration and viscous and electrical dissipation. In a very thin, suspended film with two free surfaces, the result is a $2 \mathrm{D}$ electroconvective instability, beyond which the fluid flows in the plane of the film.

The instability we consider is in principle possible in any isotropic, thin film of conducting fluid with a potential difference applied across it. To realize this experimentally, we use thin suspended films of a liquid crystal in the smectic A phase. [1, 13, 14, 15, 16, 17, These submicron films are newtonian and isotropic for flows within the plane of the film, are immune to thickness variation, and have a very low electrical conductivity. The latter is required to keep the onset voltage within an experimentally reasonable range. The fact that convection occurrs in a smectic liquid crystaline material is irrelevant except insofar as this enforces its two-dimensionality. A similar instability has been observed in suspended drops of other non-smectic fluids 24, but these are much thicker and tend to experience large thickness variations when flowing, and so are much less 2D. It is also possible to convect other, more complex, smectic phases which are anisotropic in the film plane. 25, 26] We will not consider such anisotropy here.
The system we consider consists of a thin film confined in the annular region between concentrically placed plane circular electrodes, as shown in Fig. 1. In the theory, both the film and the plate electrodes lie in the $z=0$ plane, and are treated as 2D surfaces. A voltage difference is applied to the electrodes which define the inner and outer edges of the annulus. Experimentally, we find, in the absence of Couette shear, that the bifurcation from the quiescent and conducting state to the convecting state results in a flow pattern which consists of stationary, counter-rotating pairs of vortices arranged around the circumference of the annulus. [1] Each pair of vortices has reflection symmetry and the overall pattern is spatially periodic and 1D in the azimuthal direction.

We alter the structure and symmetry of the quiescent base state by applying a Couette shear by rotating the inner electrode, as shown in Fig. 11. The shear flow leads to a net mean flow in the azimuthal direction, but unlike the previously studied cases of an imposed through-flow [4, 11, 18, the mean flow here is closed on itself. In 2D, the Couette flow by itself is stable 20,21, so that shearing alone does not introduce other instabilities. Experimentally, we find that the bifurcation from the Couette and conducting state to the convecting state leads to a $1 \mathrm{D}$ pattern which travels azimuthally. [1] When this pattern is viewed in a co-rotating frame, that is, a frame in which the pattern is stationary, we observe that it is comprised of asymmetric counter-rotating vortex pairs. The vortices with circulation in the same sense as the rotation of the inner edge are narrower than those with the opposite sense. We will show below that these features of the nonlinear flow patterns are already present in the unstable linear modes that we find from our stability analysis.

An important effect of the Couette flow is to suppress the onset of electroconvection. [1] The Couette base state is stabler against the radial electrical forcing than the unsheared state. We will show that this additional stability is a feature of the shear and not of rotation. The central testable result of the linear stability analysis we present here is the degree of this suppression for various rates of shear. We directly compare this with our experimental results obtained with annular smectic films and find satisfactory agreement. In addition, the linear stability analysis predicts the most unstable azimuthal mode and its rate of travel around the annulus.

A crucial and somewhat subtle aspect of the phenomenon is the manner in which the electrical driving force couples with the fluid. Although the fluid motion is confined to a plane, the form of the electric fields exterior to the plane, in the empty space above and below the film, are essential. These exterior fields are required for the self-consistent determination of the charge densities and thereby the electrical force. Since the electric potential at any point depends on the charge density everywhere, the coupling of the two is inherently nonlocal. This is in sharp contrast to the case of RBC, in which the coupling of the density and buoyancy body force is entirely local. [2] 
In Section II, we develop our physical model of electroconvection in a conducting $2 \mathrm{D}$ fluid. This treatment is a generalization of that presented in Ref [2]. The base state solution of these electrohydrodynamic equations in an annular geometry is presented in Section III. Fortunately, we find that the base state configuration of potentials and charges can be solved analytically in the annular geometry. The electrostatic configuration is independent of the Couette shear which forms the base flow. The shear is imposed by applying the appropriate boundary conditions on the flow velocity at the inner edge of the annulus.

The linear stability equations of the sheared base state for non-axisymmetric perturbations are set up in Section IV. Unlike for the base state itself, we find that no completely analytic solution exists of the electrostatic equations for the perturbed quantities. In the next two sections, we present two methods for solving the 3D, nonlocal coupling of the potentials and charges. In Section V, we make a local approximation to the electrostatic problem which is then used to close the perturbation equations. This yields approximate but useful analytic expressions for all of the field variables and the neutral stability boundary. In Section V1, we relax the local approximation and use a numerical scheme to solve the electrostatic equations. These results are exact, up to the accuracy of the numerical scheme. We find that the local approximation does very well over most of the parameter ranges of interest. For both solution schemes, we observe that shear suppresses the onset of convection and drives down the value of the azimuthal mode number of the convection pattern.

In Section VII, we review our experimental apparatus for convecting and shearing liquid crystal films [1], and compare the predictions of the previous sections with experiment. All of the material parameters of the fluid are known, or can be deduced from the data, so that the theoretical results for the suppression of the onset of convection can be compared to the data without adjustable parameters. We find good agreement. In Section VIII, we compare our results to what is known about other standard instabilities, particularly RBC, under shear and/or rotation. Section $\mathrm{IX}$ is a brief summary and conclusion.

\section{THE GOVERNING EQUATIONS}

In this section, our physical model describing electroconvection in a suspended thin film is presented. Our analysis here is similar to that of Ref [2]. Unless otherwise stated, all operators, field variables, and material parameters are $2 \mathrm{D}$ quantities.

The film is treated as a $2 \mathrm{D}$ conducting fluid in the $z=0$ plane, with areal density $\rho$, molecular viscosity $\eta$, and conductivity $\sigma$. We consider incompressible fluids, so that the $2 \mathrm{D}$ velocity field, $\mathbf{u}$, is divergence free,

$$
\nabla \cdot \mathbf{u}=0
$$

The Navier-Stokes equation with an electrical body force,

$$
\rho\left[\frac{\partial \mathbf{u}}{\partial t}+(\mathbf{u} \cdot \nabla) \mathbf{u}\right]=-\nabla P+\eta \nabla^{2} \mathbf{u}+q \mathbf{E},
$$

governs the fluid flow, where $\nabla, P, q$, and $\mathbf{E}$ are the 2D gradient operator, pressure field, surface charge density, and electric field in the film plane, respectively. The term $q \mathbf{E}$ is the electric force acting on the surface charge density. The charge continuity equation

$$
\frac{\partial q}{\partial t}=-\nabla \cdot(q \mathbf{u}+\sigma \mathbf{E})
$$

takes into account the convective and conductive current densities, $q \mathbf{u}$ and $\sigma \mathbf{E}$ respectively. Diffusion of charge in the plane of the film is neglected.

Subscript three will be used to denote threedimensional (3D) differential operators, material parameters and field variables. The $3 \mathrm{D}$ electric potential $\Psi_{3}$ is governed by the 3D Laplace equation,

$$
\nabla_{3}^{2} \Psi_{3}=0
$$

where $\nabla_{3}$ is the $3 \mathrm{D}$ gradient operator. The coupling of $\Psi_{3}$ with the $2 \mathrm{D}$ charge density $q$ is specified by requiring $\Psi_{3}$ to satisfy certain boundary conditions on the $z=0$ plane. The surface charge density $q$ depends on the discontinuity in the $z$-derivative of $\Psi_{3}$ across the two surfaces of the film:

$$
\begin{aligned}
q & =-\left.\epsilon_{0} \frac{\partial \Psi_{3}}{\partial z}\right|_{z=0^{+}}+\left.\epsilon_{0} \frac{\partial \Psi_{3}}{\partial z}\right|_{z=0^{-}}, \\
& =-\left.2 \epsilon_{0} \frac{\partial \Psi_{3}}{\partial z}\right|_{z=0^{+}},
\end{aligned}
$$

where $\epsilon_{0}$ is the permitivity of free space. If $q$ is known, Eqs. 2.5 constitute Neumann conditions on $\Psi_{3}$ on the film, while Dirichlet conditions, described below, hold on the electrodes. If instead Dirichlet conditions are specified on the film, Eqs. 2.5 can be used to determine $q$.

The $2 \mathrm{D}$ and $3 \mathrm{D}$ potentials are related via $\Psi=\left.\Psi_{3}\right|_{z=0}$. Equations 2.5 relate the surface charge density to the discontinuity in the $z$ component of the $3 \mathrm{D}$ electric field $\mathbf{E}_{3}=-\nabla_{3} \Psi_{3}$ across the film plane. On the other hand, the $x$ and $y$ components of $\mathbf{E}_{3}$ which form the 2D electric field $\mathbf{E}=-\nabla \Psi$, are continuous across the film. This continuity is required by the usual matching conditions for electric fields across the surfaces of dielectrics. Note that it is the $2 \mathrm{D}$ quantity $\mathbf{E}$ and not $\mathbf{E}_{3}$ that appears in equations 2.2 and 2.3. One cannot simply use a Maxwell equation to eliminate the charge density in favor of the field [27] because the 2D quantities in question are confined to a plane embedded in a 3D, otherwise empty, space and in general $\nabla \cdot \mathbf{E} \neq q / \epsilon_{0}$.

Equations 2.1 2.5. together with the appropriate boundary conditions on the electrodes, model our system. We consider the electrohydrodynamic limit where 
magnetic fields and the resultant Lorentz forces are negligible. One can also show that dielectric polarization effects are negligible in the limit of a thin film. [2]

In our subsequent analysis, we define the stream function $\phi$ by

$$
\mathbf{u}=\nabla \times \vec{\phi}
$$

where $\vec{\phi}=\phi \hat{\mathbf{z}}$. Using Eq. 2.6, $\mathbf{E}=-\nabla \Psi$ and eliminating the pressure field by applying the curl operator, Eqs. 2.2 and 2.3 reduce to

$$
\begin{aligned}
& \rho\left[\frac{\partial}{\partial t}+(\nabla \times \vec{\phi}) \cdot \nabla\right](\nabla \times \nabla \times \vec{\phi}) \\
& -\eta \nabla^{2}(\nabla \times \nabla \times \vec{\phi})+(\nabla q \times \nabla \Psi)=0,
\end{aligned}
$$

and

$$
\frac{\partial q}{\partial t}+(\nabla \times \vec{\phi}) \cdot \nabla q-\sigma \nabla^{2} \Psi=0 .
$$

We render these equations dimensionless by rescaling the length, time, and electric potential by $d, \epsilon_{0} d / \sigma$, and $V$, respectively, where $d$ and $V$ are the cross-film width and potential difference. It follows that the stream function and charge density are nondimensionalized by $\sigma d / \epsilon_{0}$ and $\epsilon_{0} V / d$. Applying this rescaling to Eqs. 2.7, 2.8, 2.4, and 2.5 gives

$$
\begin{aligned}
& {\left[\nabla^{2}-\frac{1}{\mathcal{P}} \frac{\partial}{\partial t}\right](\nabla \times \nabla \times \vec{\phi})+\mathcal{R}(\nabla \Psi \times \nabla q)=} \\
& \frac{1}{\mathcal{P}}((\nabla \times \vec{\phi}) \cdot \nabla)(\nabla \times \nabla \times \vec{\phi}), \\
& \frac{\partial q}{\partial t}+(\nabla \times \vec{\phi}) \cdot \nabla q-\nabla^{2} \Psi=0 \\
& \nabla_{3}^{2} \Psi_{3}=0, \\
& q=-\left.2 \frac{\partial \Psi_{3}}{\partial z}\right|_{z=0^{+}}
\end{aligned}
$$

where, the dimensionless parameters

$$
\mathcal{R} \equiv \frac{\epsilon_{0}^{2} V^{2}}{\sigma \eta}=\frac{\epsilon_{0}^{2} V^{2}}{\sigma_{3} \eta_{3} s^{2}} \quad \text { and } \quad \mathcal{P} \equiv \frac{\epsilon_{0} \eta}{\rho \sigma d}=\frac{\epsilon_{0} \eta_{3}}{\rho_{3} \sigma_{3} s d},
$$

are analogous to the Rayleigh and Prandtl numbers in the Rayleigh-Bénard problem. Here, $s$ is the thickness of the film and our $2 \mathrm{D}$ treatment assumes that $s \ll d$. The 2D material parameters are related to their threedimensional counterparts by $\sigma=\sigma_{3} s, \eta=\eta_{3} s$, and $\rho=\rho_{3} s$. The control parameter $\mathcal{R}$ is proportional to the square of the applied voltage difference, but independent of the film width $d$. $\mathcal{P}$ is the ratio of the charge relaxation time scale in a film $\epsilon_{0} d / \sigma_{3} s$ to the viscous relaxation time scale $\rho_{3} d^{2} / \eta_{3}$.

Equations 2.9 2.12, together with appropriate boundary conditions, describe electroconvection in a thin conducting film suspended in otherwise empty space, for any $2 \mathrm{D}$ arrangement of the film and electrodes.

\section{THE BASE STATE}

We now specify the governing equations introduced in Section II to the case of an annular film, and solve them for the case of a general Couette shear flow which forms the potentially unstable base state.

We employ cylindrical coordinates $(r, \theta, z)$ as in Fig. 1. The film is suspended between two circular electrodes which cover the remainder of the $z=0$ plane. The inner electrode has a radius $r_{i}$ and is at potential 1 in our dimensionless units. The outer electrode, which occupies the $z=0$ plane for $r>r_{o}$, is at zero potential. The cross-film width is $r_{o}-r_{i}=1$ in dimensionless units and we define the radius ratio,

$$
\alpha=r_{i} / r_{o}
$$

so that

$$
r_{i}=\frac{\alpha}{1-\alpha}, \quad r_{o}=\frac{1}{1-\alpha} .
$$

Rotation of the inner electrode about the central $r=0$ axis produces a Couette shear in the base state. We will denote base state variables by the superscript zero. Under shear, the radial derivative of base state stream function is given by

$$
\partial_{r} \phi^{(0)}(r)=\frac{\alpha^{2} \Omega}{1-\alpha^{2}}\left(r-\frac{1}{r(1-\alpha)^{2}}\right)
$$

where $\Omega$ is the dimensionless angular rotation frequency of the inner electrode. If the fluid is not sheared, the base state velocity field is zero and $\phi^{(0)}(r) \equiv 0$. The strength of the shear is described by a Reynolds number, with the velocity determined by the motion of the inner edge and the length by the film width,

$$
\mathcal{R} e=\frac{r_{i} \Omega}{\mathcal{P}}
$$

where $\mathcal{P}$ is the Prandtl number given by Equation 2.13.

There is no loss of generality by treating only rotations of the inner electrode. Since our system is $2 \mathrm{D}$, one can always reduce independent rotations of both electrodes to this case by transforming to a rotating reference frame in which the outer edge is stationary. The transformation introduces a coriolis term into Eq. 2.2, which can simply be absorbed into the pressure. [6] As a result there are important differences in the stability of $2 \mathrm{D}$ and $3 \mathrm{D}$ systems, and in particular for rigid rotation. We will return to this point in section VIII below.

The base state potential $\Psi_{3}^{(0)}(r, z)$ and charge density $q^{(0)}(r)$ are independent of the base state shear flow. They are determined by the electrostatic boundary value problem given by Eqs. 2.11 and 2.12, with Dirichlet boundary conditions on the $z=0$ plane 
$\Psi^{(0)}(r)=\Psi_{3}^{(0)}(r, 0)=\left\{\begin{array}{cc}1 & 0 \leq r \leq r_{i} \\ \frac{1}{\ln (\alpha)}[\ln (1-\alpha)+\ln (r)] & r_{i} \leq r \leq r_{o} \\ 0 & r \geq r_{o} .\end{array}\right.$

The boundary condition for $r_{i} \leq r \leq r_{o}$ is found by treating the annular film as a $2 \mathrm{D}$ ohmic conductor subject to a dimensionless potential of 1 at the inner edge and 0 at the outer, and requiring the continuity of the $2 \mathrm{D}$ current density. The logarithmic form follows from the cylindrical geometry.

The Laplace equation 2.11 for the potential in the halfspace $z>0$ is solved by the ansatz

$$
\Psi_{3}^{(0)}(r, z)=\int_{0}^{\infty} d k e^{-k z} J_{0}(k r) A(k)
$$

where $J_{0}$ is the zeroth order Bessel function. Inversion of the above equation results in

$$
A(k)=k \int_{0}^{\infty} d r r \Psi_{3}^{(0)}(r, 0) J_{0}(k r)
$$

Hence, the base state surface charge density is given by

$$
q^{(0)}(r)=2 \int_{0}^{\infty} d k k^{2} J_{0}(k r)\left[\int_{0}^{\infty} d \zeta \zeta \Psi_{3}^{(0)}(\zeta, 0) J_{0}(k \zeta)\right]
$$

where $\zeta$ is a dummy integration variable. Evaluating the integrals [28,29], we find

$$
q^{(0)}(r)=\frac{2}{\ln \alpha}\left[\frac{1}{r} F\left(\frac{1}{2}, \frac{1}{2} ; 1 ; \frac{r_{o}{ }^{2}}{r^{2}}\right)-\frac{1}{r_{i}} F\left(\frac{1}{2}, \frac{1}{2} ; 1 ; \frac{r^{2}}{r_{i}{ }^{2}}\right)\right],
$$

where $F$ is the hypergeometric function ${ }_{2} F_{1}$. This function is plotted in Fig. 2 for two values of $\alpha$. As $\alpha \rightarrow 1$, $q^{(0)}$ approaches the base state charge density for a laterally unbounded rectangular film, which is odd-symmetric about the midline of the film. This symmetry helped simplify the analysis in that case. [2] However, for $0<\alpha<1$, the annular base state charge density $q^{(0)}$ is neither even nor odd about the midline, so the analysis here is more complicated. This deviation from odd symmetry is larger for smaller $\alpha$.

The surface charge density shown in Fig. 2 is "inverted" in the sense that the positive charges lie close to the positive, inner electrode and negative charges are near the outer electrode. This unstable surface charge configuration gives rise to an electroconvective instability, much like the unstably stratified density configuration which produces Rayleigh-Bénard convection. [2] The divergences of $q^{(0)}$ at the edges of the film are a consequence of the idealized geometry in which the electrodes have zero thickness.

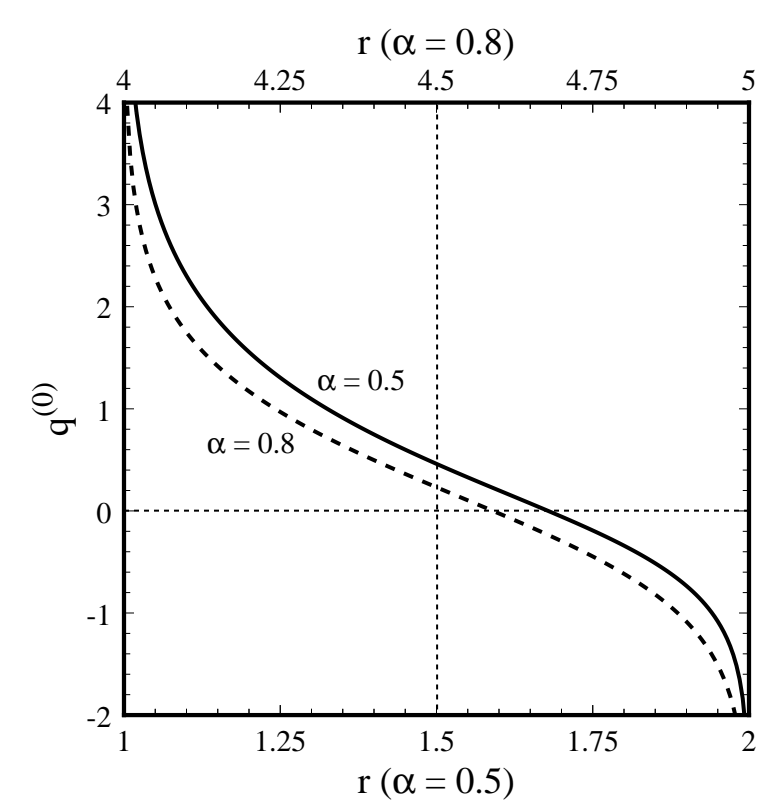

FIG. 2. Base state charge density $q^{(0)}(r)$ at $\alpha=0.5$ and $\alpha=0.8 . q^{(0)}$ diverges at the edges of the film.

This idealization leads to the boundary conditions in Eq. (3.5), whose radial derivative is discontinuous at $r=r_{i}$ and $r=r_{o}$. This is reflected in Eq. 3.9 where $F\left(\frac{1}{2}, \frac{1}{2} ; 1 ; 1\right)$ is indeterminate, so that $q^{(0)}$ diverges at the edges of the film.

\section{LINEAR STABILITY ANALYSIS}

In this section, we test the stability of the axisymmetric base state to non-axisymmetric perturbations. The perturbed quantities will be denoted by the superscript one, and are defined by

$$
\begin{aligned}
\phi(r, \theta) & =\phi^{(0)}(r)+\phi^{(1)}(r, \theta), \\
q(r, \theta) & =q^{(0)}(r)+q^{(1)}(r, \theta), \\
\Psi(r, \theta) & =\Psi^{(0)}(r)+\Psi^{(1)}(r, \theta), \\
\Psi_{3}(r, \theta, z) & =\Psi_{3}^{(0)}(r, z)+\Psi_{3}^{(1)}(r, \theta, z) .
\end{aligned}
$$

Substitution of the perturbed field variables into Eqs. 2.9 2.12 and retaining only the terms which are linear in the perturbed quantities yields

$$
\begin{array}{r}
{\left[\nabla^{2}-\frac{1}{\mathcal{P}}\left(\frac{\partial}{\partial t}-\frac{1}{r} \frac{\partial \phi^{(0)}}{\partial r} \frac{\partial}{\partial \theta}\right)\right]\left(\nabla^{2} \phi^{(1)}\right)} \\
-\frac{\mathcal{R}}{r}\left(\frac{\partial \Psi^{(0)}}{\partial r} \frac{\partial q^{(1)}}{\partial \theta}-\frac{\partial \Psi^{(1)}}{\partial \theta} \frac{\partial q^{(0)}}{\partial r}\right)= \\
\frac{1}{r \mathcal{P}} \frac{\partial \phi^{(1)}}{\partial \theta} \frac{\partial}{\partial r}\left(\frac{\partial^{2}}{\partial r^{2}}+\frac{1}{r} \frac{\partial}{\partial r}\right) \phi^{(0)}
\end{array}
$$




$$
\begin{array}{r}
\frac{\partial q^{(1)}}{\partial t}+\frac{1}{r}\left(\frac{\partial \phi^{(1)}}{\partial \theta} \frac{\partial q^{(0)}}{\partial r}-\frac{\partial q^{(1)}}{\partial \theta} \frac{\partial \phi^{(0)}}{\partial r}\right) \\
-\nabla^{2} \Psi^{(1)}=0 \\
\nabla_{3}^{2} \Psi_{3}^{(1)}=0 \\
q^{(1)}=-\left.2 \frac{\partial \Psi_{3}^{(1)}}{\partial z}\right|_{z=0^{+}}
\end{array}
$$

where

$$
\nabla^{2} \equiv \frac{\partial^{2}}{\partial r^{2}}+\frac{1}{r} \frac{\partial}{\partial r}+\frac{1}{r^{2}} \frac{\partial^{2}}{\partial \theta^{2}} \quad \text { and } \quad \nabla_{3}^{2} \equiv \nabla^{2}+\frac{\partial^{2}}{\partial z^{2}} .
$$

The variables $\phi^{(1)}, \Psi^{(1)}$, and $\Psi_{3}^{(1)}$ satisfy the following boundary conditions:

$$
\begin{gathered}
\phi^{(1)}\left(r_{i}, \theta\right)=\partial_{r} \phi^{(1)}\left(r_{i}, \theta\right)= \\
\phi^{(1)}\left(r_{o}, \theta\right)=\partial_{r} \phi^{(1)}\left(r_{o}, \theta\right)=0, \\
\Psi^{(1)}\left(r_{i}, \theta\right)=\Psi^{(1)}\left(r_{o}, \theta\right)=0 \\
\Psi_{3}^{(1)}(r, \theta, z) \rightarrow 0 \text { for } z \rightarrow \pm \infty .
\end{gathered}
$$

Equation 4.6 is a consequence of rigid boundary conditions on the fluid flow. The Dirichlet boundary conditions for the perturbed potential $\Psi_{3}^{(1)}$ on the $z=0$ plane are

$$
\Psi_{3}^{(1)}(r, \theta, 0)=\left\{\begin{array}{cl}
0 & \text { for } 0 \leq r \leq r_{i} \\
\Psi^{(1)}(r, \theta) & \text { for } r_{i} \leq r \leq r_{o} \\
0 & \text { for } r \geq r_{o} .
\end{array}\right.
$$

We decompose the perturbations into products of the following form,

$$
\left(\begin{array}{c}
\phi^{(1)}(r, \theta) \\
q^{(1)}(r, \theta) \\
\Psi^{(1)}(r, \theta) \\
\Psi_{3}^{(1)}(r, \theta, z)
\end{array}\right)=\left(\begin{array}{c}
\phi_{m}(r) \\
q_{m}(r) \\
\Psi_{m}(r) \\
\Psi_{3 m}(r, z)
\end{array}\right) e^{i m \theta+\gamma t}
$$

where the azimuthal mode number $m$ is an integer which corresponds to the number of vortex pairs in the pattern. The functions $\phi_{m}, \Psi_{m}$, and $\Psi_{3 m}$ satisfy the same boundary conditions as the perturbations, Eqs. 4.6 -4.9. The growth rate $\gamma$ may be complex.

Substitution of Eq. 4.10 into Eqs. 4.2. 4.5 gives

$$
\begin{array}{r}
\left(D_{*} D-\frac{m^{2}}{r^{2}}\right)^{2} \phi_{m} \\
-\frac{1}{\mathcal{P}}\left(\gamma-\frac{i m D \phi^{(0)}}{r}\right)\left(D_{*} D-\frac{m^{2}}{r^{2}}\right) \phi_{m} \\
-\frac{i m \mathcal{R}}{r}\left(\left(D \Psi^{(0)}\right) q_{m}-\left(D q^{(0)}\right) \Psi_{m}\right)= \\
\frac{i m \phi_{m}}{r \mathcal{P}} D\left(D_{*} D \phi^{(0)}\right), \\
\left(D_{*} D-\frac{m^{2}}{r^{2}}\right) \Psi_{m}-\left(\frac{i m D q^{(0)}}{r}\right) \phi_{m} \\
-\left(\gamma-\frac{i m D \phi^{(0)}}{r}\right) q_{m}=0,
\end{array}
$$

$$
\begin{gathered}
\left(D_{*} D-\frac{m^{2}}{r^{2}}+\frac{\partial^{2}}{\partial z^{2}}\right) \Psi_{3 m}=0, \\
q_{m}=-\left.2 \frac{\partial \Psi_{3 m}}{\partial z}\right|_{z=0^{+}},
\end{gathered}
$$

where $D \equiv \partial_{r}$ and $D_{*} \equiv D+1 / r$. Henceforth, we limit our discussion to a base state flow which is either quiescent or Couette. In these cases $D\left(D_{*} D \phi^{(0)}\right) \equiv 0$ and the right hand side of Eq. 4.11 is identically zero. [20] It can be shown that in the limit $\alpha \rightarrow 1$, and for zero shear $\phi^{(0)} \equiv 0$, Eqs. 4.114 .14 reduce to the linear stability equations for electroconvection in a laterally unbounded strip. [2]

We write the complex growth exponent $\gamma$ as $\gamma^{r}+i \gamma^{i}$. In order to find the conditions for marginal stability, we set the real part $\gamma^{r}=0$. Our task is then to solve Eqs. 4.11 4.14 for a given $\alpha, \mathcal{P}$ and $\mathcal{R} e$, by determining consistent values of $\mathcal{R}$ and $\gamma^{i}$ for each $m$. The rate of azimuthal travel of each marginally stable mode around the annulus is $\gamma^{i} / m$. The marginal stability boundary, which is defined only at discrete $m$, has a minimum at the critical values $m_{c}, \mathcal{R}_{c}$, while the critical mode travels at $\gamma_{c}^{i} / m_{c}$.

We employ the following basic expansions:

$$
\begin{aligned}
\phi_{m}(r) & =\sum_{n} A_{n} \phi_{m ; n}(r), \\
\Psi_{m}(r) & =\sum_{n} A_{n} \Psi_{m ; n}(r), \\
\Psi_{3 m}(r, z) & =\sum_{n} A_{n} \Psi_{3 m ; n}(r, z), \\
q_{m}(r) & =\sum_{n} A_{n} Q_{m ; n}(r),
\end{aligned}
$$

where the $A_{n}$ are amplitudes. The expansion eigenfunctions $\phi_{m ; n}(r), \Psi_{m ; n}(r)$, and $\Psi_{3 m ; n}(r, z)$ satisfy the following boundary conditions:

$$
\begin{gathered}
\phi_{m ; n}\left(r_{i}\right)=\partial_{r} \phi_{m ; n}\left(r_{i}\right)= \\
\phi_{m ; n}\left(r_{o}\right)=\partial_{r} \phi_{m ; n}\left(r_{o}\right)=0, \\
\Psi_{m ; n}\left(r_{i}\right)=\Psi_{m ; n}\left(r_{o}\right)=0 \\
\Psi_{3 m ; n}(r, z) \rightarrow 0 \text { for } z \rightarrow \pm \infty \\
\Psi_{3 m ; n}(r, z=0)=\left\{\begin{array}{ccc}
0 & \text { for } 0 \leq r \leq r_{i} \\
\Psi_{m ; n}(r) & \text { for } & r_{i} \leq r \leq r_{o} \\
0 & \text { for } & r \geq r_{o}
\end{array} .\right.
\end{gathered}
$$

The functions $\phi_{m ; n}(r)$, which satisfy the 'rigid' boundary conditions given in Eq. 4.19, can be identified with the Chandrasekhar cylinder functions [7],

$$
\begin{array}{r}
\phi_{m ; n}(r)=\mathcal{C}_{m ; n}(r)=J_{m}\left(\beta_{m n} r\right)+B_{m n} Y_{m}\left(\beta_{m n} r\right) \\
+C_{m n} I_{m}\left(\beta_{m n} r\right)+D_{m n} K_{m}\left(\beta_{m n} r\right) .
\end{array}
$$

The boundary conditions Eq. 4.20, imply that the 2D potential expansion function $\Psi_{m ; n}$ can be further expanded in a series of functions of the form, 


$$
\psi_{m ; p}(r)=J_{m}\left(\chi_{m p} r\right)+b_{m p} Y_{m}\left(\chi_{m p} r\right) .
$$

The functions $\mathcal{C}_{m ; n}$ and $\psi_{m ; p}$, along with their associated constants $\beta_{m n}, B_{m n}, C_{m n}, D_{m n}, \chi_{m p}$, and $b_{m p}$ are described in detail in the Appendix.

In the next two sections, we use these expansions to solve Eqs. 4.11-4.14, first approximately and then in general.

\section{LINEAR STABILITY: LOCAL APPROXIMATION}

The main barrier to solving Eqs. 4.11 4.14 lies in the difficult nonlocal coupling between $\Psi_{m}$ and $q_{m}$ in Eqs. 4.11 and 4.12 which is required by the $3 \mathrm{D}$ electrostatic equations 4.13 and 4.14. In this section, we circumvent this problem by making an approximation. This yields expressions which give some insight into the general linear stability problem.

To make the approximation, we replace the 3D Eqs. 4.13 and 4.14 with the following simple 2D closure relation:

$$
\left(D_{*} D-\frac{m^{2}}{r^{2}}\right) f_{m} \Psi_{m}=-q_{m} .
$$

In the above expression, $f_{m}$ is a closure factor which is independent of $r$, and is to be specified. As we show below, a consequence of this approximation is that the charge density and the $2 \mathrm{D}$ potential are related pointwise, or locally.

This approximation is motivated by following physical reasoning. If instead of an annular film, one considers an annular column, with a height much larger than its width, then there is a straightforward Poisson relation between a bulk charge density and the 3D potential inside the column. If the $3 \mathrm{D}$ potential is independent of $z$, and is equal to $\Psi_{m}(r) e^{i m \theta}$, there are no free surfaces to consider and one has in place of Eqs. 4.13 and 4.14 the relation $\left(D_{*} D-m^{2} / r^{2}\right) \Psi_{m}=-q_{m}$. If one now hypothesizes that the charge density retains its radial profile when the bulk is 'squeezed down' to a 2D film, then one must include only an $r$ independent scaling factor, $f_{m}$, as in Eq. 5.1.

We make use of 4.16 and further expand each $\Psi_{m ; n}$ using Eq. 4.24 so that

$$
\Psi_{m}=\sum_{n} A_{n} \Psi_{m ; n}=\sum_{n} A_{n} \sum_{p} B_{m ; n p} \psi_{m ; p},
$$

where the $B_{m ; n p}$ are constants. It then follows from Eqs. 5.1 and Eq. A9 that

$$
\begin{array}{r}
q_{m}=\sum_{n} A_{n} \sum_{p} B_{m ; n p} f_{m ; p} \chi_{m p}{ }^{2} \psi_{m ; p}= \\
\sum_{n} A_{n} \sum_{p} B_{m ; n p} q_{m ; p},
\end{array}
$$

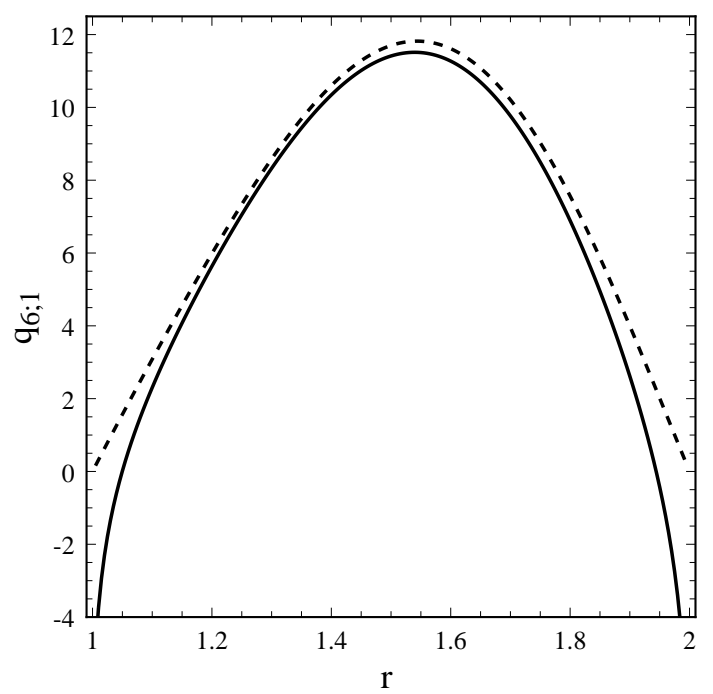

FIG. 3. The charge density perturbation $q_{6 ; 1}(r)$ at $\alpha=0.50$, as determined by the local approximation of Section $\mathrm{V}$ (dashed line) and the nonlocal solution of Section VI (solid line). The nonlocal result diverges to $-\infty$ at the edges of the film.

where

$$
q_{m ; p}=f_{m ; p} \chi_{m p}^{2} \psi_{m ; p},
$$

which demonstrates the pointwise, local relation between the potential on the film and its surface charge density in this approximation.

A choice for $f_{m ; p}$ can be made by considering the empty upper half space $z>0$ with homogeneous boundary conditions at infinity and Dirichlet boundary conditions such that the potential is equal to $\psi_{m ; p}(r) e^{i m \theta}$ everywhere on the $z=0$ plane. This boundary condition is smooth, unlike the piecewise smooth conditions in Eq. 4.22, which respect the sharp edges of the annulus. Then the 3D potential for $z \geq 0$ satisfies the equation,

$$
\left(D_{*} D-\frac{m^{2}}{r^{2}}+\frac{\partial^{2}}{\partial z^{2}}\right) \psi_{m ; p} e^{-k_{m p} z}=0 .
$$

Using the eigenvalue relation Eq. A9, it follows that $k_{m p}=\chi_{m p}$. The corresponding surface charge density is thus

$$
q_{m ; p}=-\left.2 \frac{\partial}{\partial z} \psi_{m ; p} e^{-\chi_{m p} z}\right|_{z=0^{+}}=2 \chi_{m p} \psi_{m ; p} .
$$

Comparing Eq. 5.6 with Eq. 5.4 leads to the following choice for the closure factor:

$$
f_{m ; p}=\frac{2}{\chi_{m p}} .
$$

For the remainder of this section, we adopt this method of closing the equations. Fig. 3 shows a plot of the approximate charge density, $q_{6 ; 1}$ at $\alpha=0.5$, corresponding to a 
potential $\psi_{6 ; 1}$ on the film. It is compared to a more accurate numerical solution discussed in Section VI, below. As might be expected, the approximation is accurate except close to the edges of the film.

We can now solve the remaining $2 \mathrm{D}$ equations using the expansions in Eqs. 4.15, 4.23, 5.2 and 5.3. We consider the simplest case: a single expansion mode, so that $A_{1}=$ 1 and $A_{n}=0$ for $n>1$. Similarly, we truncate the expansions of the potential and charge density at $p=1$ so that $B_{m ; 1 p} \equiv 0$ for $p>1$. The expansion coefficient $B_{m} \equiv B_{m: 11}$ is complex. Thus, using Eq. 5.7, Eqs. 4.15. 5.2 and 5.3 reduce to

$$
\begin{aligned}
\phi_{m} & =\mathcal{C}_{m ; 1}, \\
\Psi_{m} & =\left(B_{m}^{r}+i B_{m}^{i}\right) \psi_{m ; p}, \\
q_{m} & =2\left(B_{m}^{r}+i B_{m}^{i}\right) \chi_{m 1} \psi_{m ; 1} .
\end{aligned}
$$

Substitution of Eq. 5.8 into Eq.4.12, with $\gamma=i \gamma^{i}$ results in an equation, the real and imaginary parts of which are

$$
\begin{array}{r}
B_{m}^{r} \chi_{m 1} \psi_{m ; 1}=2\left(\gamma^{i}-\frac{m D \phi^{(0)}}{r}\right) B_{m}^{i} \psi_{m ; 1} \\
B_{m}^{i} \chi_{m 1}^{2} \psi_{m ; 1}+2\left(\gamma^{i}-\frac{m D \phi^{(0)}}{r}\right) B_{m}^{r} \chi_{m 1} \psi_{m ; 1} \\
+\left(\frac{m D q^{(0)}}{r}\right) \mathcal{C}_{m ; 1}=0
\end{array}
$$

respectively. Eliminating $B_{m}^{r}$ from the above pair of equations results in

$$
\begin{array}{r}
{\left[1+\frac{4}{\chi_{m 1^{2}}}\left(\gamma^{i}-\frac{m D \phi^{(0)}}{r}\right)^{2}\right] B_{m}^{i} \chi_{m 1}^{2} \psi_{m ; 1}} \\
+\left(\frac{m D q^{(0)}}{r}\right) \mathcal{C}_{m ; 1}=0
\end{array}
$$

Multiplying by $\psi_{m ; 1}$, and integrating to form inner products,

$$
\langle\ldots\rangle=\int_{r_{i}}^{r_{o}} \ldots r d r
$$

we solve for the expansion constant,

$$
B_{m}^{i}=\frac{-m}{\chi_{m 1}^{2} \mathcal{N}_{\psi_{m ; 1}}} \mathbf{L}_{m}
$$

where $\mathcal{N}_{\psi_{m ; 1}}$ is a normalization factor given in the Appendix and the matrix element

$$
\begin{aligned}
\mathbf{L}_{m} & =\left\langle\mathcal{C}_{m ; 1} \frac{D q^{(0)}}{r}\right. \\
& \times\left[1+\frac{4}{\chi_{m 1^{2}}}\left(\gamma^{i}-\frac{m D \phi^{(0)}}{r}\right)^{2}\right]^{-1} \\
& \left.\times \psi_{m ; 1}\right\rangle
\end{aligned}
$$

A similar projection of Eq. 5.9 and some simplification results in

$$
B_{m}^{r}=\frac{2 B_{m}^{i}}{\chi_{m 1}}\left(\gamma^{i}-\frac{m}{\mathcal{N}_{\psi_{m ; 1}}}\left\langle\psi_{m ; 1} \frac{D \phi^{(0)}}{r} \psi_{m ; 1}\right\rangle\right) .
$$

Equations 5.13 5.15 determine the expansion coefficients of the potential and charge density for a given stream function. Substitution of Eq. 5.8 into Eq. 4.11, with $\gamma=$ $i \gamma^{i}$ and a Couette shear gives

$$
\begin{array}{r}
\beta_{m 1}{ }^{4} \mathcal{C}_{m ; 1}-i \frac{\beta_{m 1}{ }^{2}}{\mathcal{P}}\left(\gamma^{i}-\frac{m D \phi^{(0)}}{r}\right)\left(\mathcal{V}_{m ; 1}-\mathcal{U}_{m ; 1}\right) \\
-i \frac{m \mathcal{R}}{r}\left(( B _ { m } ^ { r } + i B _ { m } ^ { i } ) \left(2 \chi_{m 1} D \Psi^{(0)}\right.\right. \\
\left.\left.-D q^{(0)}\right) \psi_{m ; 1}\right)=0
\end{array}
$$

where $\mathcal{U}_{m ; 1}$ and $\mathcal{V}_{m ; 1}$ are defined in the Appendix. Projecting the above equation with $C_{m ; 1}$ allows us to solve for $\mathcal{R}$ :

$$
\begin{array}{r}
\mathcal{R}\left(\alpha, \mathcal{P}, \mathcal{R} e, m, \gamma^{i}\right)= \\
\frac{\beta_{m 1}{ }^{4} \mathcal{N}_{\mathcal{C}_{m ; 1}}+i \mathcal{P}^{-1} \beta_{m 1}^{2}\left(m \mathbf{F}_{m}-\gamma^{i} \mathbf{G}_{m}\right)}{m\left(i B_{m}^{r}-B_{m}^{i}\right)\left(2 \chi_{m 1} \mathbf{J}_{m}-\mathbf{K}_{m}\right)},
\end{array}
$$

where

$$
\begin{aligned}
\mathbf{F}_{m} & =\left\langle\mathcal{C}_{m ; 1} \frac{D \phi^{(0)}}{r}\left(\mathcal{V}_{m ; 1}-\mathcal{U}_{m ; 1}\right)\right\rangle, \\
\mathbf{G}_{m} & =\left\langle\mathcal{C}_{m ; 1}\left(\mathcal{V}_{m ; 1}-\mathcal{U}_{m ; 1}\right)\right\rangle, \\
\mathbf{J}_{m} & =\left\langle\mathcal{C}_{m ; 1}\left(\frac{D \Psi^{(0)}}{r}\right) \psi_{m ; 1}\right\rangle, \\
\mathbf{K}_{m} & =\left\langle\mathcal{C}_{m ; 1}\left(\frac{D q^{(0)}}{r}\right) \psi_{m ; 1}\right\rangle .
\end{aligned}
$$

The normalization factor $\mathcal{N}_{\mathcal{C}_{m ; 1}}$ is given in the Appendix.

To determine the linear stability boundary for a given radius ratio $\alpha$, Prandtl number $\mathcal{P}$ and Reynolds number $\mathcal{R} e$, we solve Eq. 5.17 for a sequence of azimuthal mode numbers $m$, using Mathematica for all integrations. We first consider some special cases.

For zero shear, $\phi^{(0)}=\gamma^{i}=0$ and Eq. 5.17 reduces to

$$
\mathcal{R}(\alpha, m)=\frac{\beta_{m 1}{ }^{4} \chi_{m 1}{ }^{2} \mathcal{N}_{\mathcal{C}_{m ; 1}} \mathcal{N}_{\psi_{m ; p 1}}}{m^{2} \mathbf{K}_{m}\left(2 \chi_{m 1} \mathbf{J}_{m}-\mathbf{K}_{m}\right)}
$$

Note that Eq. 5.22 is independent of the Prandtl number and is always real. Fig. A shows the resulting stability boundary for $\alpha=0.5$. Except for being discretized in integer values of $m$ by the annular geometry, the boundary is not much different than that of the infinite rectangular 


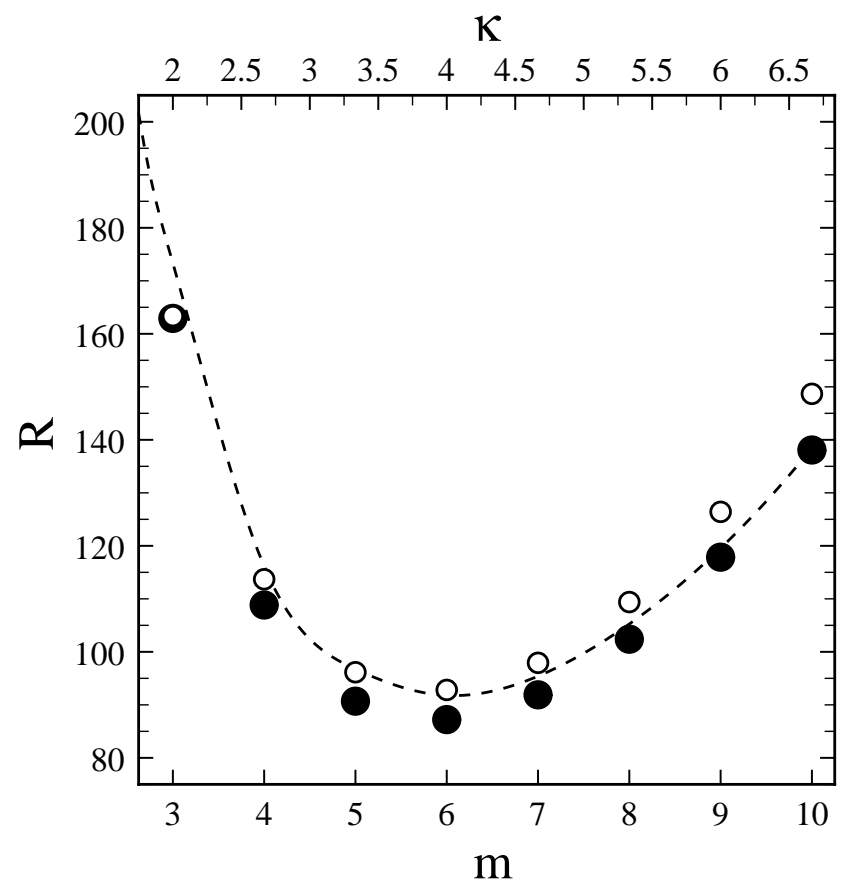

FIG. 4. The marginal stability boundary $\mathcal{R}$ vs. $m$ for zero applied shear and $\alpha=0.5$. Open symbols show the result from the local approximation (Section $\mathrm{V}$ ), , solid symbols show the full nonlocal stability calculation (Section VI), with three expansion modes. The dashed line indicates the continuous marginal stability curve for the unbounded rectangular geometry with plate electrodes (see Ref. [2]). The azimuthal mode number $m$ is equivalent to a dimensionless wavenumber $\kappa=m / \bar{r}$ (upper scale), where $\bar{r}$ is the mean radius $\left(r_{i}+r_{o}\right) / 2$.

case [2], which is defined at a continuum of wavevectors $\kappa$. The discrete curve approaches the continuum one in the limit $\alpha \rightarrow 1$. Also shown in Fig. 4 is the more accurate numerical solution presented in Section V1, below. The minima of this curve define the critical parameters $m_{c}^{0}(\alpha) \equiv m_{c}(\alpha, \mathcal{R} e=0)$ and $\mathcal{R}_{c}^{0}(\alpha) \equiv \mathcal{R}_{c}(\alpha, \mathcal{R} e=0)$. Some values for various $\alpha$ are collected in Table I.

For non-zero shear, Eq. 5.17 simplifies somewhat in the limit that the Prandtl number $\mathcal{P} \rightarrow \infty$. A word of caution is perhaps appropriate here. As $\mathcal{P} \rightarrow \infty$, we require $\Omega \rightarrow \infty$ such that $\Omega / \mathcal{P}$ and therefore $\mathcal{R} e$ (see Eq. 3.4) are finite. In this limit, with the proviso that $\mathcal{R}$ be real, Eq. 5.17 becomes

$$
\mathcal{R}\left(\alpha, \mathcal{R} e, m, \gamma^{i}\right)=\frac{\beta_{m 1}{ }^{4} \chi_{m 1}{ }^{2} \mathcal{N}_{\mathcal{C}_{m ; 1}} \mathcal{N}_{\psi_{m ; p 1}}}{m^{2} \mathbf{L}_{m}\left(2 \chi_{m 1} \mathbf{J}_{m}-\mathbf{K}_{m}\right)}
$$

The only shear dependence in Eq. 5.23 occurs through the matrix element $\mathbf{L}_{m}$, which is defined by Eq. 5.14. For zero shear, $\mathbf{L}_{m} \equiv \mathbf{K}_{m}$, while for non-zero shear, $\mathbf{L}_{m}$ is bounded above by $\mathbf{K}_{m}$. Hence, $\mathcal{R}\left(\alpha, \mathcal{R} e, m, \gamma^{i}\right)$ is bounded below by $\mathcal{R}\left(\alpha, \mathcal{R} e=0, m, \gamma^{i}=0\right)$, the zero shear value. Thus, one expects suppression of the onset of convection for non-zero shear for every non-axisymmetric mode $m$. This feature is also present for finite $\mathcal{P}$.

\begin{tabular}{|c|c|c|c|c|c|c|c|}
\hline $\begin{array}{l}\text { radius } \\
\text { ratio }\end{array}$ & \multicolumn{2}{|c|}{$\begin{array}{l}\text { local } \\
(n=1, p=1)\end{array}$} & \multicolumn{2}{|c|}{$\begin{array}{l}\text { nonlocal } \\
(n=1, l=20)\end{array}$} & \multicolumn{3}{|c|}{$\begin{array}{l}\text { nonlocal } \\
(n=3, l=20)\end{array}$} \\
\hline$\alpha$ & $m_{c}^{0}$ & $\mathcal{R}_{c}^{0}$ & $m_{c}^{0}$ & $\mathcal{R}_{c}^{0}$ & $m_{c}^{0}$ & $m_{c}^{0} / \bar{r}$ & $\mathcal{R}_{c}^{0}$ \\
\hline 0.33 & 4 & 102.58 & 4 & 9162 & 4 & \begin{tabular}{|l|}
4.03 \\
\end{tabular} & 82.15 \\
\hline & & & & & & & 89.6 \\
\hline & & & & & & & 91.1 \\
\hline 0.80 & 18 & 8.84 & 19 & 93.59 & 19 & 4.22 & 93.1 \\
\hline
\end{tabular}

TABLE I. Critical parameters for zero shear as determined by the local approximation (Section $\mathrm{V}$ ) and the full nonlocal (Section VI) linear stability analysis. The integer $n(p$ or $l$ ) is the number of modes (expansion functions) used in the series representation of the field variables. The critical wavevector for electroconvection in a laterally unbounded geometry is $\kappa_{c}=4.223$ (see Ref. [2]). The ratio $m_{c}^{0} / \bar{r} \rightarrow \kappa_{c}$ as $\alpha \rightarrow 1$, where $\bar{r}$ is the mean radius $\left(r_{i}+r_{o}\right) / 2$.

\begin{tabular}{|c|c|c|c|c|c|c|c|}
\hline \hline Prandtl & $\begin{array}{l}\text { Rotation } \\
\text { rate }\end{array}$ & \multicolumn{2}{l}{$\begin{array}{l}\text { local } \\
(n=1, p=1)\end{array}$} & \multicolumn{3}{l|}{$\begin{array}{l}\text { nonlocal } \\
(n=1, l=20)\end{array}$} \\
\hline $\mathcal{P}$ & $\Omega$ & $m_{c}$ & $\mathcal{R}_{c}$ & $\gamma_{c}^{i}$ & $m_{c}$ & $\mathcal{R}_{c}$ & $\gamma_{c}^{i}$ \\
\hline 100 & 5.5135 & 8 & 319.50 & -14.949 & 8 & 282.61 & -16.383 \\
10 & 0.5513 & 9 & 99.65 & -1.655 & 9 & 98.52 & -1.861 \\
1 & 0.0551 & 9 & 90.16 & -0.166 & 10 & 93.06 & -0.205 \\
0.1 & 0.0055 & 9 & 90.05 & -0.017 & 10 & 93.00 & -0.020 \\
0.01 & 0.0006 & 9 & 90.04 & -0.002 & 10 & 93.00 & -0.002 \\
\hline \hline
\end{tabular}

TABLE II. Critical Parameters for a range of Prandtl numbers $\mathcal{P}$ at radius ratio $\alpha=0.6446$ and Reynolds number $\mathcal{R} e=0.1$. The integer $n$ ( $p$ or $l$ ) is the number of modes (expansion functions) used in the series representation of the field variables.

\begin{tabular}{|c|c|c|c|c|c|c|c|}
\hline Prandtl & Reynolds & $\begin{array}{l}\text { loca } \\
(n=\end{array}$ & $1, p=$ & & $\begin{array}{l}\text { non } \\
(n=\end{array}$ & $\begin{array}{l}\text { ocal } \\
=1, l=2\end{array}$ & \\
\hline $\mathcal{P}$ & $\operatorname{Re}$ & $m_{c}$ & $\mathcal{R}_{c}$ & $\overline{\gamma_{c}^{\imath}}$ & $m_{c}$ & $\mathcal{R}_{c}$ & $\overline{\gamma_{c}^{2}}$ \\
\hline 100 & 0.0181 & 9 & 115.20 & -2.995 & 9 & 109.03 & -3.372 \\
\hline 10 & 0.1814 & 9 & 115.29 & -3.002 & 9 & 109.02 & -3.365 \\
\hline 1 & 1.8137 & 9 & 115.27 & -3.007 & 9 & 108.99 & -3.307 \\
\hline 0.1 & 18.1373 & 9 & 115.24 & -3.039 & 9 & 109.14 & -3.165 \\
\hline 0.01 & 181.3731 & 9 & 115.16 & -3.086 & 9 & 109.31 & -3.108 \\
\hline
\end{tabular}

TABLE III. Critical Parameters for a range of Prandtl numbers $\mathcal{P}$ at radius ratio $\alpha=0.6446$ and rotation rate $\Omega=1.0$. The integer $n(p$ or $l)$ is the number of modes (expansion functions) used in the series representation of the field variables. 
For arbitrary $\alpha, \mathcal{P}$ and $\mathcal{R} e$, one can solve Eq. 5.17 for various $m$ by a $1 \mathrm{D}$ search procedure. At each $m, \gamma^{i}$ is varied to find a $\mathcal{R}$ that is real and a minimum. Example neutral curves are shown in Fig. 5 for $\alpha=0.8, \mathcal{P}=10$ and several $\mathcal{R} e$. The suppression of convective onset is evident, as well as a tendency for the critical mode number $m_{c}$ to decrease with $\mathcal{R} e$. We find that $\mathcal{R}(\mathcal{R} e)$ is a monotonically increasing function of $\mathcal{R} e$. The dependence of $\mathcal{R}$ on $\mathcal{P}$ is treated in two ways. We will first fix $\mathcal{R} e$, change $\mathcal{P}$ and consequently let $\Omega$ vary. In the second protocol we will fix $\Omega$, change $\mathcal{P}$ and consequently let $\mathcal{R} e$ vary. For fixed $\mathcal{R} e$, the $\mathcal{P}$ dependence of these results is not strong, except at large $\mathcal{P}$. Because $\mathcal{R} e$ is proportional to $\mathcal{P}^{-1}$ in Eq. 3.4, this limit corresponds to large values of $\Omega$ and a large suppression effect. Some results for various $\mathcal{P}$ are summarized in Table II For fixed $\Omega$, the $\mathcal{P}$ dependence is very weak at all $\mathcal{P}$ investigated. Results are summarized in Table III.

\section{LINEAR STABILITY: EXACT NONLOCAL SOLUTION}

We now relax the local approximation described in the previous section, and properly treat the full problem. The electrostatic Eqs. 4.13-4.14, in which the charge density and electrostatic potential are related nonlocally, are solved numerically. As well as being more physically correct, this allows us to determine the accuracy of the local approximation.

The first step in the present method is to find the appropriate expansion functions of the field variables. Substitution of Eqs. 4.15 4.18 into Eqs. 4.12 4.14 yields equations which can be solved for $A_{n}, \phi_{m ; n}, \Psi_{m ; n}, \Psi_{3 m ; n}$, and $Q_{m ; n}$. The stream function $\phi_{m ; n}(r)=C_{m ; n}(r)$ as in Eq. 4.23. The potentials $\Psi_{m ; n}$ and $\Psi_{3 m ; n}$ and the charge density $Q_{m ; n}$ are further expanded as

$$
\begin{aligned}
\Psi_{m ; n}(r) & =\sum_{l} v_{m ; n l} \psi_{m ; l}(r), \\
\Psi_{3 m ; n}(r) & =\sum_{l} v_{m ; n l} \psi_{3 m ; l}(r), \\
Q_{m ; n}(r) & =\sum_{l} v_{m ; n l} q_{m ; l}(r),
\end{aligned}
$$

where $\psi_{m ; l}$ is given by Eq. 1.24 and $v_{m ; n l}$ are complex coefficients. The functions $\psi_{3 m ; l}$ and $q_{m ; l}$ are computed as follows. After substituting Eqs. 4.17 and 6.2 into Eq. 4.13, the resulting equation

$$
\left(D_{*} D-\frac{m^{2}}{r^{2}}+\frac{\partial^{2}}{\partial z^{2}}\right) \psi_{3 m ; l}=0,
$$

is solved numerically on a finite $2 \mathrm{D}$ grid by an overrelaxation algorithm [30] for the functions $\psi_{3 m: l}(r, z)$, subject to the boundary conditions as in Eqs. $4.21-4.22$ with $\psi_{3 m ; l}(r, 0)=\psi_{m: l}(r)$ for $r_{i} \leq r \leq r_{o}$ and 0 otherwise. Then Eqs. 4.14, 4.17, 4.18, and 6.2-6.4 give

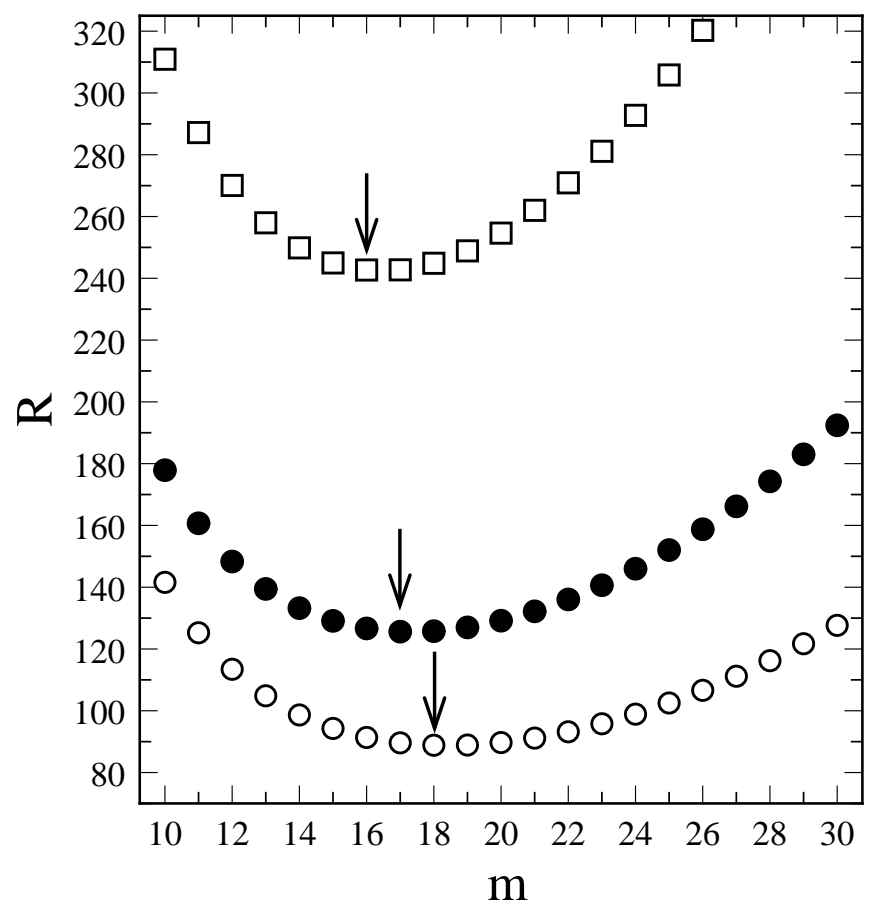

FIG. 5. Marginal stability boundaries under shear, showing $\mathcal{R}$ as a function of $m$ for $\alpha=0.80$ and $\mathcal{P}=10$, using the local approximation (Section $\mathrm{V}$ ). Shown are the boundaries for Reynolds numbers $\mathcal{R} e=0.0$ (circles), 0.25 (filled circles) and 0.75 (squares). Arrows indicate the critical mode.

$$
q_{m ; l}(r)=-\left.2 \partial_{z} \psi_{3 m ; l}(r, z)\right|_{z=0^{+}},
$$

where the differentiation is performed numerically. Fig. 3 shows a plot of the charge density $q_{6 ; 1}$ at $\alpha=0.5$, and compares the numerical result to the approximate one given by Eqs. 5.4 and 5.7 from the previous section. The approximate solution does not contain the divergences which occur near the film's edges, due to the sharp changes in the derivative of the potential. These are a feature of our 2D model, which treats the film and electrodes as having zero thickness.

We next substitute Equations 6.1 and 6.3 into Eq. 4.12 and use

$$
C_{m ; n}\left(\frac{D q^{(0)}}{r}\right)=\sum_{l}\left\langle C_{m ; n}\left(\frac{D q^{(0)}}{r}\right) \psi_{m ; l}\right\rangle \psi_{m ; l}
$$

The resulting equation is projected against $\psi_{m ; k}$ to obtain a matrix expression that can be solved numerically for the complex coefficients $v_{m ; n l}$.

Finally, substituting the various expansions in $\psi_{m ; l}$ into Eq. 4.11 and taking the inner product with $C_{m ; p}$, yields a set of linear homogeneous equations for the constants $A_{n}$. We write this set as the matrix equation $\sum_{n} A_{n} \mathbf{T}_{p n}=0$. For a nontrivial solution, the compatibility condition is 


$$
\operatorname{Real}(\operatorname{det}[\mathbf{T}])=\operatorname{Imag}(\operatorname{det}[\mathbf{T}])=0,
$$

with the elements of the matrix $\mathbf{T}$ given by

$$
\begin{array}{r}
\mathbf{T}_{p n}=\left\langle C_{m ; p}\left(D_{*} D-\frac{m^{2}}{r^{2}}\right)^{2} C_{m ; n}\right\rangle \\
-\frac{i}{\mathcal{P}}\left\langle C_{m ; p}\left(\gamma^{i}-m\left(\frac{D \phi^{(0)}}{r}\right)\right)\left(D_{*} D-\frac{m^{2}}{r^{2}}\right) C_{m ; n}\right\rangle \\
-i m \mathcal{R} \mathbf{F}_{m ; p n} .
\end{array}
$$

The first two inner products of Eq. 6.8 can be simplified using Eqs. A1 and $\mathrm{A} 8$ and the matrix elements $\mathbf{F}_{m ; p n}$ are

$$
\mathbf{F}_{m ; p n}=\sum_{l} v_{m ; n l}\left\langle\frac{C_{m ; p}}{r}\left(\left(D \Psi^{(0)}\right) q_{m ; l}-\left(D q^{(0)}\right) \psi_{m ; l}\right)\right\rangle .
$$

The real values of $\mathcal{R}$ and $\gamma^{i}$ which satisfy Eqs. 6.7 and 6.8 at each $m$ define the neutral stability boundary $\mathcal{R}=$ $\mathcal{R}\left(\alpha, \mathcal{P}, \mathcal{R} e, m, \gamma^{i}\right)$. The critical parameters $m_{c}, \mathcal{R}_{c}$ and $\gamma_{c}^{i}$ are obtained when $\mathcal{R}$ is minimized.

The numerical over-relaxation calculation used to solve Eq. 6.4 involved a grid spacing such that there was a minimum of 160 points across the width of the film. For the purposes of integration, the discrete values of $q_{m ; l}$ found numerically from Eq. 6.5 were Chebyshev interpolated. The series in Eqs. 6.1 6.3 and 6.6 were calculated up to $l=20$. Three modes $(n=p=3)$ were employed in the compatibility conditions Eq. 6.7 when the shear was zero. This was reduced to one mode $(n=p=1)$ when the shear was non-zero. All $r$-integrations were performed by the Romberg method. [30 We solved Real $(\operatorname{det}[\mathbf{T}])=0$ (Eq. 6.7) for $\mathcal{R}$ for a given trial $\gamma^{i}$ and associated coefficients $v_{m ; n l}$ of the field variables. The $\mathcal{R}$ and trial $v_{m ; n l}$ were employed in the search for the $\gamma^{i}$ which satisfied $\operatorname{Imag}(\operatorname{det}[\mathbf{T}])=0$. The new value of $\gamma^{i}$ was then used to find new coefficients $v_{m: n l}$. The iterative cycle was continued until the parameters and field variables were converged.

The marginal stability boundary for $\alpha=0.5$ and zero shear is shown in Fig. 4 , using both the local approximations and the full nonlocal solution. Neither solution is qualitatively much different from the infinite rectangular case. [2] For the nonlocal solution, we find that as $\alpha \rightarrow 1, m_{c}^{0}$ increases such that $m_{c}^{0} / \bar{r}$ approaches the correct limiting value, $\kappa_{c}=4.223$. Here, $\bar{r}=\left(r_{i}+r_{o}\right) / 2$ is the midline radius of the annulus and $\kappa_{c}$ is the critical wavenumber for an infinite rectangular film in the "plate" electrode geometry. [2] Table [I shows a sample set of critical parameters as determined by both solution schemes. There is generally good agreement between the two methods, except at small $\alpha$, where more expansion modes are needed.

Fig. 6 shows the zero shear critical control parameter $\mathcal{R}_{c}^{0}$ as a function of the radius ratio $\alpha$. There are discrete values of $\alpha$ where $\mathcal{R}_{c}^{0}\left(\alpha, m_{c}\right)=\mathcal{R}_{c}^{0}\left(\alpha, m_{c}+1\right)$, so that

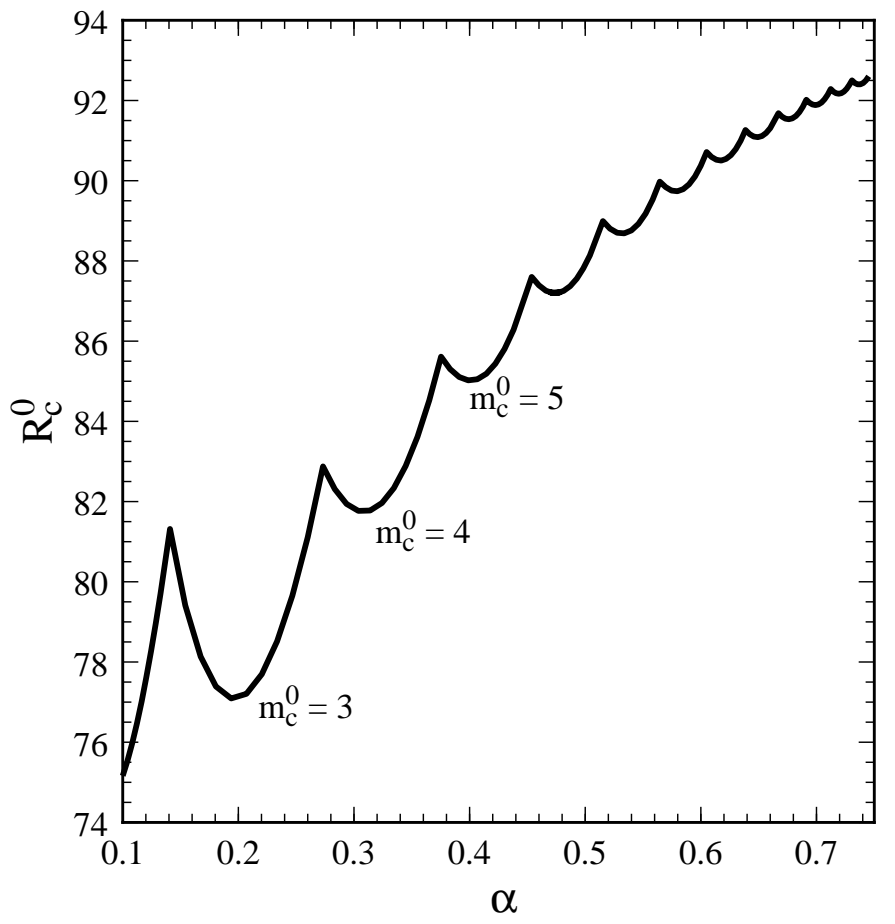

FIG. 6. The critical control parameter for zero shear $\mathcal{R}_{c}^{0}$ versus radius ratio $\alpha$ using the nonlocal stability analysis with three expansion modes. Special radius ratios, at which two adjacent modes $m$ and $m+1$ are simultaneously marginally unstable, occur at the cusps of the curve. Between cusps, the critical mode number $m_{c}^{0}$ remains constant.

two adjacent modes become unstable simultaneously at a co-dimension two point. These points occur at the cusps in Fig. 6, while between the cusps a single value of $m$ is critical. It is interesting to note that the trend in $\mathcal{R}_{c}^{0}$ is increasing overall, as function of $\alpha$. This is opposite to what is found for radially driven Rayleigh-Bénard convection in a rotating annulus. [6] We attribute this difference as a manifestation of the differences between the nature of the charge density 'inversion' and thereby electrical forcing with the bouyancy inversion of RBC. As $\alpha \rightarrow 1$, the co-dimension two points become closely spaced and the value of $\mathcal{R}_{c}^{0}$ approaches a limiting value. 38

For the case of non-zero shear, the nonlocal analysis produces neutral curves which resemble those shown in Fig. 5 . For fixed $\alpha$ and $\mathcal{P}$, the critical control parameter $\mathcal{R}_{c}$ increases with $\mathcal{R} e$, which indicates a suppression of convection as is observed in experiments. [1] This prediction is compared to experimental data in Section VII below. The $\mathcal{P}$ dependence of $\mathcal{R}_{c}$ for $\mathcal{R} e \neq 0$ is rather weak at small $\mathcal{P}$. This is outlined in Tables II and III. Note that for fixed $\mathcal{R} e$, large $\mathcal{P}$ implies large $\Omega$ and thus greater stabilization. For fixed $\Omega$, however, the stability propoerties are insensitive to $\mathcal{P}$ and therefore to $\mathcal{R} e$.

The critical mode number $m_{c}$ decreases with increasing shear. This effect is shown in Fig. 6, which displays 


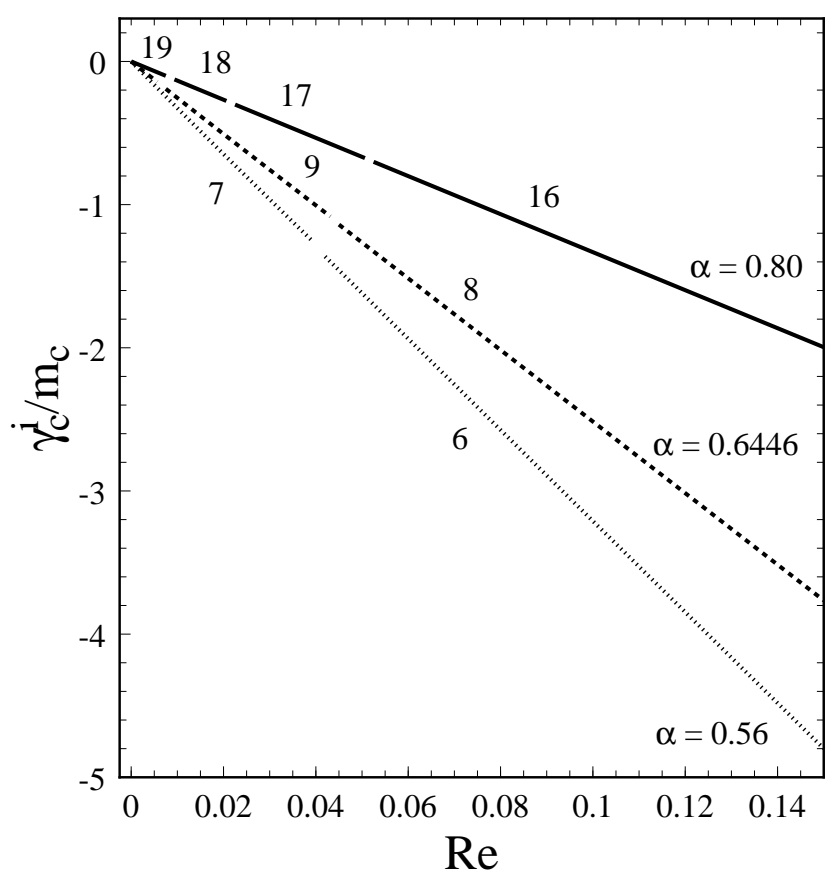

FIG. 7. Ratio of imaginary part of the growth rate to critical mode number, $\gamma_{c}^{i} / m_{c}$ versus Reynolds number $\mathcal{R} e$ for $\alpha=0.56,0.6446$, and 0.80 and $\mathcal{P}=123$ using the nonlocal linear stability calculation with one expansion mode. The breaks in the curves for each $\alpha$ show the intervals over which the critical azimuthal mode number $m_{c}$ has the value indicated.

the angular traveling rate of the critical mode, $\gamma_{c}^{i} / m_{c}$, as a function of $\mathcal{R} e$ for several $\alpha$. For $\Omega>0, \gamma_{c}^{i}<0$, which indicates that the critical mode travels around the annulus in the same sense as the inner electrode. $\gamma_{c}^{i} / m_{c}$ is a very nearly linearly decreasing function of $\mathcal{R} e$, with very small discontinuities at points where the critical azimuthal mode number $m_{c}$ changes, as shown in Fig. 7 . Each of these discontinuities, which are too small to resolve on the scale of Fig. I, is a co-dimension two point, where two adjacent $m$ modes with very slightly different traveling rates are simultaneously unstable at onset. These special points are also slightly $\mathcal{P}$ dependent, as well as being $\alpha$ dependent in a manner similar to the zero shear case discussed above.

Although linear analysis cannot provide the magnitude of the fields above onset, it is nevertheless interesting to examine the spatial structure of the linearly unstable modes. Figure 8 displays the velocity vector field of a critical mode for $\alpha=0.56$, plotted with an arbitrary amplitude. In Fig. 8(a), we show the stationary vortex pattern at $\mathcal{R} e=0$. Here $m_{c}^{0}=7$, so 7 symmetric vortex pairs are arranged around the annulus. This pattern is purely non-axisymmetric or "columnar". It is an exact solution to the governing equations and has been observed experimentally. As is discussed in Section VIII below, we would expect exactly the same pattern under the same conditions for any rate of rigid rotation. In Fig. 8(b), we show the typical flow pattern for (a)

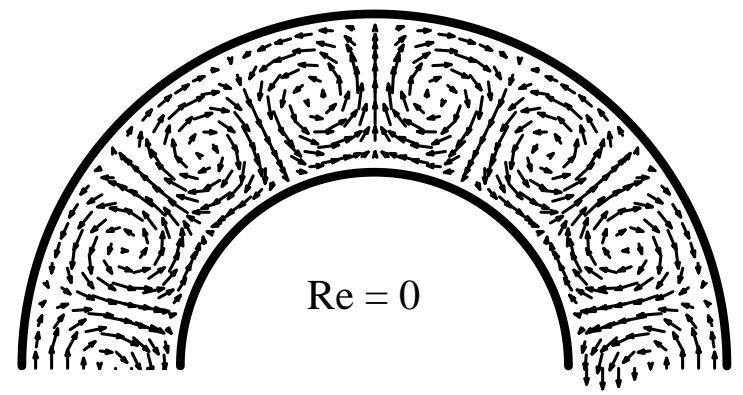

(b)

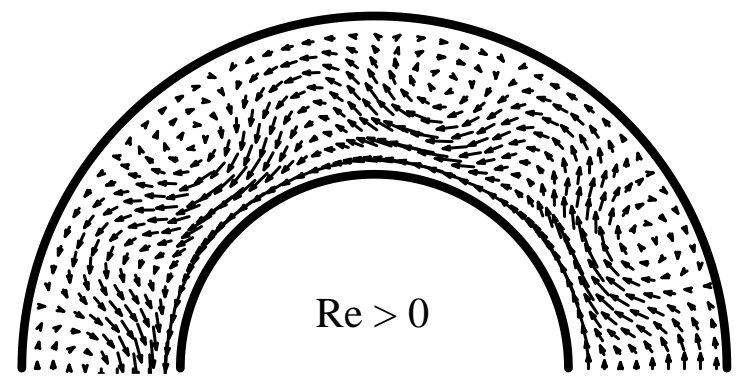

(c)

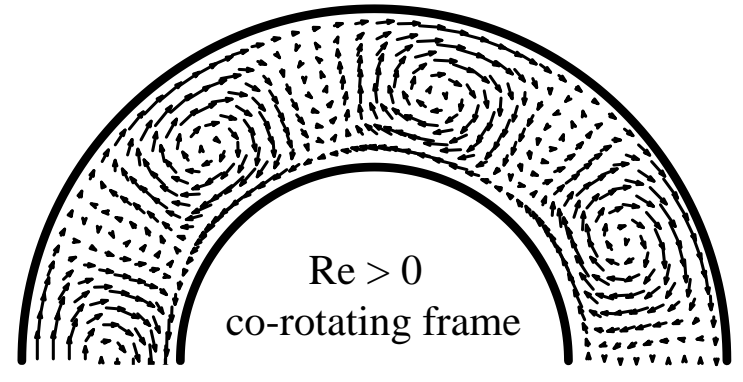

FIG. 8. The velocity vector field of arbitrary amplitude for $\alpha=0.56$ with (a) $\mathcal{R} e=0$, (b) $\mathcal{R} e>0$ as viewed in the laboratory frame, and (c) $\mathcal{R} e>0$ as seen in the frame co-rotating at $\gamma_{c}^{i} / m_{c}$ in which the pattern is stationary.

a large $\mathcal{R} e>0$, as viewed in the laboratory frame. The periodicity of the pattern is reduced (i.e. $m_{c}<m_{c}^{0}$ ), and the traveling pattern appears as a meandering wave in the laboratory frame. Fig. $8(\mathrm{c})$ shows the same $\mathcal{R} e$ as in (b), but as seen in the frame in which the pattern is stationary. This frame rotates in the same sense as the inner electrode but with an angular speed of $\gamma_{c}^{i} / m_{c}$, which is less than $\Omega$. In this frame, each vortex pair consists of a larger and a smaller member. The same qualitative features are seen in sheared convection patterns just above onset in the present experiments and those of Ref. [1].

\section{EXPERIMENT}

In this section, we discuss our experimental investigation of the linear stability of 2D fluids to electroconvection and Couette shear. The apparatus we describe is only slightly modified from that previously reported in 
Ref [1]. Our objective here is to experimentally test one of the direct predictions of the linear theory, namely the suppression of onset by shear.

A smectic A liquid crystal (octylcyanobiphenyl, or $8 \mathrm{CB}$ ) was employed to realize a $2 \mathrm{D}$ fluid. In the smectic A phase, long organic molecules arrange themselves into layers with their long axes normal to the layer. Within the layer planes, smectic A behaves as a $2 \mathrm{D}$ isotropic fluid, and can be drawn into robust submicron films which are integer numbers of layers thick and uniform over their whole area. 13. 31 Other smectic phases form 2D anisotropic fluid films. 31,25,26] Unlike soap films, smectics can flow in the film plane without thickness change and are not susceptible to evaporation. Smectics generally have low electrical conductivity due to residual ionic impurities. 31,32] This combination of properties makes smectic films uniquely suited for studying electroconvection. While our experiments used liquid crystal films, the electroconvective instability itself is generic and not specific to anisotropic fluids; a similar instability is found in thin drops or puddles of ordinary isotropic fluids. [24, 33, 34] The anisotropy introduced by the smectic layering simply serves to constrain the film to have a constant, very small, thickness. It behaves isotropically for flows in the plane of interest.

Our experimental apparatus consisted of two concentrically placed stainless steel electrodes which supported a suspended film. The inner electrode was a disk of radius $3.61 \pm 0.01 \mathrm{~mm}$ and the outer electrode was a plate with a circular hole of radius $6.40 \pm 0.01 \mathrm{~mm}$, giving an annular film with radius ratio $\alpha=0.564 \pm 0.002$. Each molecular layer of smectic A $8 \mathrm{CB}$ is $3.16 \mathrm{~nm}$ thick. The inner electrode could be rotated about its axis by a high precision stepper motor at angular frequencies up to $4 \mathrm{rad} / \mathrm{s}$, while the outer was fixed. All experiments were performed at $23 \pm 1^{\circ} \mathrm{C}$. The air surrounding the film was pumped down to an ambient pressure of 0.5-5 torr. The low pressure ensures that air drag on the film will be insignificant. [35] The system was electrically shielded by a Faraday cage and the outer electrode and shield were maintained at ground potential while the voltage $V$ of the inner electrode was varied above ground. The resulting current through the film was measured using a picoammeter. Except for a few qualitative runs to observe the vortex patterns, we did not visualize the flow, as this required suspending particles in the film, which was not necessary for our present purposes. The film behaved as an ohmic conductor in the conduction regime below the onset of convection. The critical voltage $V_{c}$, at which the onset of convection occurred, was located from the sharp increase in current. In all runs, the shear rate was held constant while the voltage was slowly increased and decreased through onset. For some rates of shear, the onset of convection was hysteretic. [1] In these cases, we took $V_{c}$ to be the transition from conduction to convection as $V$ is increased.

In order to interpret the experimentally measured quantities, we need to accurately express them in nondi- mensional form. According to Eq. 2.13, the critical control parameter $\mathcal{R}_{c}$ is related to the critical voltage $V_{c}$ by

$$
\mathcal{R}_{c}=\frac{\epsilon_{0}^{2} V_{c}^{2}}{\sigma_{3} \eta_{3} s^{2}} .
$$

The size of the experimental uncertainties on $\sigma_{3}, s$, and especially, $\eta_{3}$ prevented us from directly using Eq. 7.1 to scale the voltages. Rather, we used the zero shear measurements of $V_{c}^{0}$ to scale the data for non-zero shear. A relative measure of the suppression of the onset of convection is

$$
\tilde{\epsilon}(\mathcal{R} e)=\left[\frac{\mathcal{R}_{c}(\mathcal{R} e)}{\mathcal{R}_{c}^{0}}\right]-1=\left[\frac{V_{c}(\mathcal{R} e)}{V_{c}^{0}}\right]^{2}-1 .
$$

The first part of Eq. 7.2 was applied to the theoretical results, while the second was applied to the experimental data. The latter scaling does not require a knowledge of the material parameters. In order to obtain the remaining dimensionless quantities, $\mathcal{R} e$ and $\mathcal{P}$, estimates of $\sigma_{3}$, $s$, and $\eta_{3}$ are required. However, we will show below that our final results are independent of $s$ and $\sigma_{3}$ and quite insensitive to the value of $\eta_{3}$.

It is straightforward to measure a quantity proportional to the product $\sigma_{3} s$ from the data. For $V<V_{c}$, the film behaves ohmically and hence the slope of the current-voltage data is the film conductance $c$, given by

$$
c=\frac{2 \pi \sigma_{3} s}{\ln (1 / \alpha)} .
$$

For a given current-voltage sweep, $c$ can be measured to within a few percent. This precision is limited by the tendency of the conductance of the material to drift over the course of the sweep. [36] Using many measured values of $c$, we found an average value of $\sigma_{3}$, which was consistent with all the data. The film thickness $s$ can be independently deduced by examining the color of the film under reflected white light. [16] By measuring $c$ and $s$ for a number of films with various $\alpha$, we found the driftaveraged conductivity to be $\sigma_{3}=1.3 \pm 0.2 \times 10^{-7}(\Omega m)^{-1}$. We used this value to infer a consistent value of $\eta_{3}$, via the procedure described below.

Eqs. 7.1 and 7.3 can be combined to give

$$
\left[\frac{2 \pi \epsilon_{0}}{\ln (1 / \alpha) \sqrt{\mathcal{R}_{c}^{0}}}\right] V_{c}^{0}=\sqrt{\frac{\eta_{3}}{\sigma_{3}}} c .
$$

To find $\eta_{3}$, we plotted the left hand side of Eq. 7.4 against measurements of $c$, using measured values of $V_{c}^{0}$ and the theoretical value of $\mathcal{R}_{c}^{0}$. We used data from a large number of films with $s<60$ smectic layers for two values of $\alpha, 0.56$ and 0.64 . A one parameter fit to find the slope of this plot established $\sqrt{\eta_{3} / \sigma_{3}}$. Using the average value of $\sigma_{3}$ given above, we found $\eta_{3}=0.16 \pm 0.06 \mathrm{~kg} / \mathrm{ms}$. This method has the advantage that it does not require an independent measurement of $s$ for every film. 


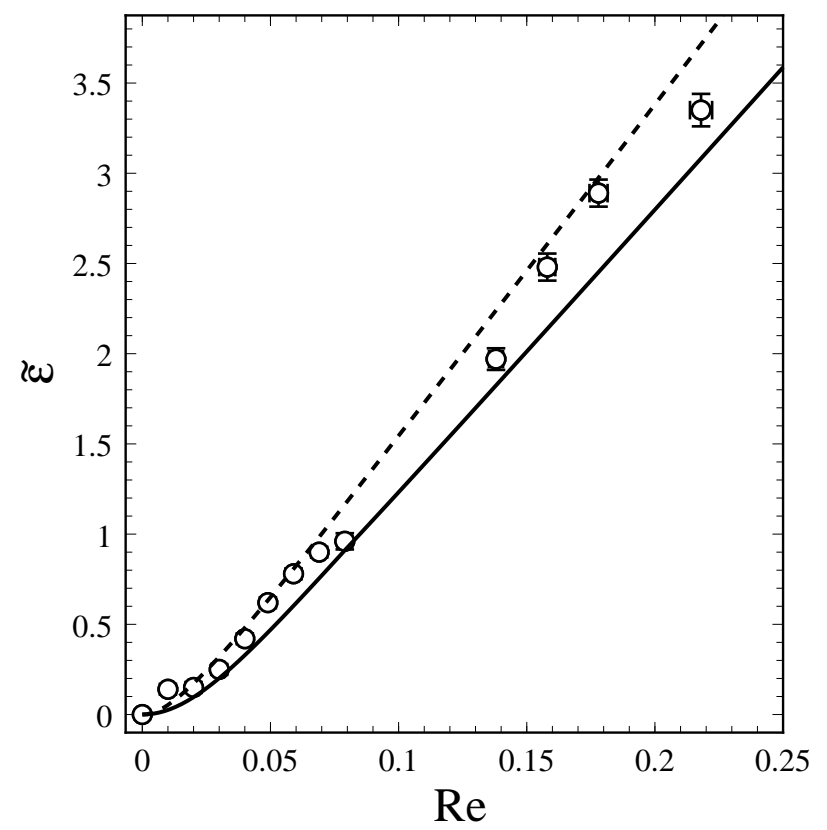

FIG. 9. Comparison of the experimental and theoretical relative suppression $\tilde{\epsilon}$ versus Reynolds number $\mathcal{R} e$ for $\alpha=0.56$. The dashed (solid) line is the result of the local (nonlocal) method.

We then employed the above value of $\eta_{3}$ to calculate $\mathcal{R} e$ which is expressed in terms of dimensional parameters by

$$
\mathcal{R} e=\rho_{3} \omega r_{i}\left(r_{o}-r_{i}\right) / \eta_{3}
$$

where $\omega$ is the measured angular frequency of the inner electrode in rad $/ \mathrm{s}$. The $3 \mathrm{D}$ density $\rho_{3}$ of the liquid crystal is 37 $1.0 \times 10^{3} \mathrm{~kg} / \mathrm{m}^{3}$. Similarly, the Prandtl number $\mathcal{P}$ is given in terms of measured quantities by

$$
\mathcal{P}=\frac{2 \pi \epsilon_{0} \eta_{3}}{\rho_{3}\left(r_{o}-r_{i}\right) \ln (1 / \alpha)} \frac{1}{c} .
$$

From the definition, Eq. 2.13, $\mathcal{P}$ depends on $s$. In Eq. 7.6, this dependence is contained in $c$, which can be accurately measured for each individual current-voltage sweep. Thus, for films of various constant thicknesses, $\mathcal{P}$ experiences a small drift from sweep to sweep, due to the drift in $c$. For the data we report here, $\mathcal{P}$ was always in the range $52<\mathcal{P}<75$. If $c$ is treated as an independent, experimentally determined variable, then Eq. 7.6 is independent of $s$ and $\sigma_{3}$.

We can now compare our experimental results and the predictions of the linear theory for non-zero shear. Fig. 9 shows the theoretical curves from the local and nonlocal calculations and the experimental data for the relative suppression $\tilde{\epsilon}$, as a function of $\mathcal{R} e$. The theory curves are plotted for a fixed $\mathcal{P}=63.5$. There are no adjustable parameters. We find good agreement between the experimental data and the linear theory for $\mathcal{R} e<0.22$. The local approximation slightly overestimates the suppression, while the exact nonlocal result slightly underestimates it. Recall that the local approximation neglects the divergence in the perturbed charge density at the edges of the film, but uses the full base state solution, which has similar divergences in our 2D model. The nonlocal calculation keeps the divergences at both orders. The fact that these two treatments bracket the data suggests that the divergences are rounded off by the finite thickness of the film and electrodes in the actual experimental geometry.

The good agreement shown in Fig. 9 is essentially independent of the value of $\eta_{3}$. Since the $\eta_{3}$ dependence in the $\mathcal{R} e$ scaling of both the theory (via $\mathcal{P}$ in Eq. 3.4) and the experiment (according to Eq. 7.5) are proportional to $1 / \eta_{3}$, any change in $\eta_{3}$ multipies both by the same factor. This simply results in a rescaling of the $\mathcal{R} e$ axis in Fig. 9, with no change in the quality of the agreement.

\section{DISCUSSION}

It is interesting to compare our results for $2 \mathrm{D}$ electroconvection under shear with those for other, more familiar instabilities under shear or rotation.

In a theoretical study, Agrait and Castellanos [19] considered electroconvection due to a radial field in a $3 \mathrm{D}$ concentric cylinder geometry with charge injection on either cylinder. Both cylinders were permitted to rotate to produce a general Couette shear. Their result was that shearing enhanced the instability, leading to a 3D flow that resembles TVF. This can easily be understood as a consequence of the fact that in $3 \mathrm{D}$, the base state Couette shear itself is unstable. In our 2D system, the shear flow is not only stable by itself [20,21], but, as we have shown, has a stabilizing effect on the electroconvection. This is one of several examples of how the $2 \mathrm{D}$ nature of our flow leads to an unusual stability, which is often difficult or impossible to realize experimentally in other systems.

The added stability in our system is a consequence of the shear and not of rotation. Under rigid rotation, where the inner and outer electrodes are co-rotating, one can transform to rotating co-ordinates in which the electrodes are stationary. This transformation introduces a coriolis term $-2 \Omega \hat{\mathbf{z}} \times \overrightarrow{\mathbf{v}}=-2 \Omega \nabla \psi$ in Eqn. 2.2 which may be absorbed into the pressure gradient term $\nabla P$ and eliminated. [6] Thus, in a purely 2D system, rigid rotation and the non-rotating, unsheared case have identical stability. It also follows, since the transformation is general and the unsheared bifurcation is stationary, that the resulting nonlinear vortex pattern above onset must be stationary in the co-rotating frame.

This lack of dependence under rigid rotation may be contrasted with a large class of 3D and quasi-2D rotating Rayleigh-Bénard systems [5, 8, 9, 10], where rotation produces added stability but the absence of strictly $2 \mathrm{D}$ flow results in time-dependence (precession) of the convection pattern in the co-rotating frame. Chandrasekhar [7] treated the classic problem of the linear stability of $\mathrm{RBC}$ in a laterally unbounded layer rotating about its 
normal. The case of a laterally bounded cylindrical layer has received much recent interest theoretically [8], and has also been the subject of a precise experimental study [9].

A concise summary of some of the work on rotating RBC can be found in Ref. [10]. Due to the similar symmetries in these scenarios, some results are common to most rotating RBC systems. 10] Since, these systems are three-dimensional (3D), the solutions they support can generally be classified into axisymmetric, non-axisymmetric ("columnar") or mixed (combinations of axisymmetric and non-axisymmetric) solutions. The principal result is that for $3 \mathrm{D}$ mixed solutions, the onset bifurcation is no longer steady as it is for non-rotating RBC. This leads to a flow pattern that precesses in the co-rotating frame. The onset of these solutions is however suppressed, raising the critical Rayleigh number above its non-rotating value. 8,9,10] Purely non-axisymmetric or columnar solutions are strictly two-dimensional (2D). When they do occur, they do not precess but their onset occurs at the same critical Rayleigh number as in the absence of rotation. [6, 10] There have also been theoretical studies of the interesting but nonetheless experimentally unrealizable situation of $2 \mathrm{D} \mathrm{RBC}$ in a rotating annular geometry with purely radial gravity and heating [6]. These studies found similar columnar solutions. In fact, purely columnar solutions ("Taylor columns" [39]) have yet to be observed in any rotating $\mathrm{RBC}$ experiment since the boundary conditions at the top and botton of the cylinder must be stress free [6,10], a requirement that cannot be attained in terrestrial RBC experiments. In contrast, two-dimensionality, stress free end boundary conditions and radial driving forces all arise naturally in the electroconvection of an annular suspended film that we have described.

We next consider sheared, but non-rotating systems. RBC has been studied experimentally and theoretically with an open through-flow. [4,11] The throughflow is generally a weak Poiseuille flow with a very small Reynolds number. Its effects on RBC are well understood. In brief, the onset of convection is again suppressed, but the first instability is convective (i.e. it grows only downstream of a localized perturbation), rather than absolute. The resulting convection pattern drifts in the direction of the through flow. It is interesting that the clear distinction between convective and absolute instability is blurred in systems like ours, in which the "through" flow loops back on itself. As we have analyzed it, the onset of convection in our annular electroconvection is absolute.

There have been some theoretical studies of 3D RBC in the presence of a plane Couette shear flow [12], another situation which is experimentally unrealizable. Here, linear analysis reveals stability differences between transverse roll disturbances, those with axes are perpendicular to the shear, and longitudinal roll disturbances which have axes parallel to the shear flow. Longitudinal-roll disturbances have identical stability properties to RBC in the absence of shear, and are always more unstable than the transverse-roll disturbances. Transverse-roll disturbances, conversely, exhibit suppression, or added stability due to the shear. The onset Rayleigh number of transverse rolls is a monotonically increasing function of the shear Reynolds number, similar to what we find for $2 \mathrm{D}$ electroconvection. Furthermore, the critical wavenumber of the most unstable transverse disturbance was found to be a monotonically decreasing function of the shear Reynolds number, also as we observe. Transverse rolls (vortices, in fact) appear at onset in our 2D system, but would always be pre-empted by longitudinal rolls in a 3D $\mathrm{RBC}$ experiment, if it could be realized.

\section{SUMMARY AND CONCLUSION}

In this paper, we have analyzed the linear stability of a weakly conducting, two-dimensional annular fluid to radially-driven electroconvection. We considered the effects of an imposed Couette shear produced by the rotation of the inner edge of the annulus. We calculated the marginal stability boundary for the Rayleigh number $\mathcal{R}(\alpha, \mathcal{P}, \mathcal{R} e, m)$ as a function of the azimuthal mode number $m$, for general radius ratio $\alpha$, Prandtl number $\mathcal{P}$ and Reynolds number $\mathcal{R} e$. We also found the azimuthal traveling rate $\gamma^{i} / m$ of the marginally unstable modes, where $\gamma^{i}$ is the imaginary part of the linear growth rate. We found a set of discrete values of $\alpha, \mathcal{P}$ and $\mathcal{R} e$ for which there are two adjacent azimuthal modes $m$ which are simultaneously unstable at onset. Two solutions schemes were employed: a local approximation which neglected some aspects of the coupling of fields and charges, and a more exact, fully nonlocal method. These methods agreed, except in some quantitative details.

When there is no applied shear, so that $\mathcal{R} e=0$, we found $\gamma^{i}=0$ and the most unstable linear mode did not travel. As $\alpha \rightarrow 1$, the marginal stability boundary for this case approached that for a laterally unbounded rectangular film. [2] For non-zero shear, $\mathcal{R} e \neq 0$, the most unstable linear mode traveled in the direction of the rotation of the inner electrode and the onset of convection was suppressed, relative to the zero shear case.

The shear also reduced the critical azimutal mode number $m_{c}$. Rigid rotation has no effect on the critical azimuthal mode number $m_{c}$, again contrasting with the class of 3D rotating Rayleigh-Bénard systems [9] where increasing rotation rate favored higher critical azimutal mode number $m_{c}$.

We performed experiments on thin, suspended annular films of smectic A liquid crystals, which are well described by our theoretical model. We measured the current through the film as a function of the driving voltage for a fixed rotation rate of the inner electrode. This data was used to determine the onset of convection and two of the material parameters needed to scale the data. We compared the relative suppression of the onset from the 
experiments to that predicted by the linear theory and found good agreement. The spatial structure of the traveling modes from the linear theory resembled the weakly nonlinear vortex patterns observed experimentally, when viewed in a co-rotating reference frame. [1]

Due to the rich nonlinear aspects of this system, there are several interesting future directions. The system is sufficiently tractable that the weakly nonlinear regime should be accessible to theoretical investigation. It may also be interesting to simulate the nonlinear regime numerically. On the experimental side, we expect to be able to study the secondary bifurcations quantitatively, and make good connection to the nonlinear theory. It would be useful to determine the material parameters, particularly the viscosity, by separate experiments. It should be possible to study regimes of higher $\mathcal{R} e$. Finally, it would be interesting to examine other flows, such as a time-dependent oscillatory shear, superposed on electroconvection.

\section{ACKNOWLEDGMENTS}

We would like to thank John R. de Bruyn and Wayne A. Tokaruk for numerous discussions. This research was supported by the Natural Sciences and Engineering Research Council of Canada.

\section{APPENDIX A}

\section{Expansion functions for the stream function}

Since the stream function is constrained by rigid boundary conditions at $r=r_{i}$ and $r=r_{o}$ and obeys Eq. 4.11, it can be expanded in the eigenfunctions of the square of the Laplacian operator. [7] Hence, the eigenfunctions sought are defined by the eigenvalue relation

$$
\left(D_{*} D-\frac{m^{2}}{r^{2}}\right)^{2} \mathcal{C}_{m ; n}=\beta_{m n}^{4} \mathcal{C}_{m ; n}
$$

The boundary conditions are $\mathcal{C}_{m ; n}\left(r_{i}\right)=\mathcal{C}_{m ; n}\left(r_{o}\right)=0$ and $D \mathcal{C}_{m ; n}\left(r_{i}\right)=D \mathcal{C}_{m ; n}\left(r_{o}\right)=0$. The desired solutions of Eq. A1 are

$$
\begin{gathered}
\mathcal{C}_{m ; n}(r)=J_{m}\left(\beta_{m n} r\right)+B_{m n} Y_{m}\left(\beta_{m n} r\right) \\
\quad+C_{m n} I_{m}\left(\beta_{m n} r\right)+D_{m n} K_{m}\left(\beta_{m n} r\right),
\end{gathered}
$$

where $J_{m}$ and $Y_{m}$ are the familiar Bessel and Weber functions of order $m, I_{m}$ and $K_{m}$ are the modified Bessel functions of order $m$. The parameters $\beta_{m n}$ are successive solutions of the secular equation $\operatorname{det} \mathbf{M}(\beta)=0$, where

$$
\left[\begin{array}{cccc}
J_{m}\left(\beta r_{i}\right) & Y_{m}\left(\beta r_{i}\right) & I_{m}\left(\beta r_{i}\right) & K_{m}\left(\beta r_{i}\right) \\
J_{m}\left(\beta r_{o}\right) & Y_{m}\left(\beta r_{o}\right) & I_{m}\left(\beta r_{o}\right) & K_{m}\left(\beta r_{o}\right) \\
J_{m-1}\left(\beta r_{i}\right) & Y_{m-1}\left(\beta r_{i}\right) & I_{m-1}\left(\beta r_{i}\right) & -K_{m-1}\left(\beta r_{i}\right) \\
J_{m-1}\left(\beta r_{o}\right) & Y_{m-1}\left(\beta r_{o}\right) & I_{m-1}\left(\beta r_{o}\right) & -K_{m-1}\left(\beta r_{o}\right)
\end{array}\right]
$$

and $\left(1, B_{m n}, C_{m n}, D_{m n}\right)$ is the eigenvector corresponding to eigenvalue zero for each $\beta_{m n}$. We tabulated the $\beta_{m n}$ at each radius ratio $\alpha$ using a multiple root finding routine. 30 The determinant was found using an SVD algorithm, and the eigenvectors by backsubstitution. 30 It was necessary to rescale the third and fourth columns of $\mathbf{M}$ before taking the determinant to avoid underflows and overflows.

The functions defined by Eq. A2 form a complete, orthogonal set with orthogonality condition

$$
\int_{r_{i}}^{r_{o}} d r r \mathcal{C}_{m ; n} \mathcal{C}_{m ; p}=\mathcal{N}_{C_{m ; n}} \delta_{n p}
$$

The normalization $\mathcal{N}_{C_{m ; n}}$ is given by

$$
\mathcal{N}_{C_{m ; n}}=r_{o}^{2} \mathcal{U}_{m ; n}^{2}\left(r_{o}\right)-{r_{i}}^{2} \mathcal{U}_{m ; n}^{2}\left(r_{i}\right)
$$

where

$$
\mathcal{U}_{m ; n}(r)=J_{m}\left(\beta_{m n} r\right)+B_{m n} Y_{m}\left(\beta_{m n} r\right)
$$

It is convenient to define a function $\mathcal{V}_{m ; n}$ by

$$
\mathcal{V}_{m ; n}(r)=C_{m n} I_{m}\left(\beta_{m n} r\right)+D_{m n} K_{m}\left(\beta_{m n} r\right)
$$

so that $\mathcal{C}_{m ; n}(r)=\mathcal{U}_{m ; n}(r)+\mathcal{V}_{m ; n}(r)$ and

$$
\left(D_{*} D-\frac{m^{2}}{r^{2}}\right) C_{m ; n}=-\beta_{m n}^{2}\left(\mathcal{U}_{m ; n}-\mathcal{V}_{m ; n}\right)
$$

\section{Expansion functions for the potential}

The potential perturbation satisfies homogeneous boundary conditions at the inner and outer edges of the film and obeys Eq.4.12, so that we can use an expansion in terms of the eigenfunctions of the Laplacian operator,

$$
\left(D_{*} D-\frac{m^{2}}{r^{2}}\right) \psi_{m ; l}=-\chi_{m l}^{2} \psi_{m ; l},
$$

which has solutions

$$
\psi_{m ; n}(r)=J_{m}\left(\chi_{m n} r\right)+b_{m n} Y_{m}\left(\chi_{m n} r\right) .
$$

The parameters $\chi_{m n}$ solve the secular equation $\operatorname{det} \mathbf{N}(\chi)=0$, where

$$
\mathbf{N}(\chi)=\left[\begin{array}{cc}
J_{m}\left(\chi r_{i}\right) & Y_{m}\left(\chi r_{i}\right) \\
J_{m}\left(\chi r_{o}\right) & Y_{m}\left(\chi r_{o}\right)
\end{array}\right]
$$

and $\left(1, b_{m n}\right)$ is the eigenvector corresponding to eigenvalue zero for each $\chi_{m n}$. These constants were found by routines similar to the ones used in Section A1. The functions defined by Eq. A10 satisfy the orthogonality condition, 


$$
\int_{r_{i}}^{r_{o}} d r r \psi_{m ; n} \psi_{m ; p}=\mathcal{N}_{\psi_{m ; n}} \delta_{n p}
$$

where the normalization $\mathcal{N}_{\psi_{m ; n}}$ is given by

$$
\begin{aligned}
\mathcal{N}_{\psi_{m ; n}} & =\frac{1}{8}\left[r _ { o } { } ^ { 2 } \left(\left[J_{m-1}\left(\chi_{m n} r_{o}\right)-J_{m+1}\left(\chi_{m n} r_{o}\right)\right]\right.\right. \\
& +b_{m n}\left[\left(Y_{m-1}\left(\chi_{m n} r_{o}\right)-Y_{m+1}\left(\chi_{m n} r_{o}\right)\right]\right)^{2} \\
& -r_{i}{ }^{2}\left(\left[J_{m-1}\left(\chi_{m n} r_{i}\right)-J_{m+1}\left(\chi_{m n} r_{i}\right)\right]\right. \\
& \left.\left.+b_{m n}\left[Y_{m-1}\left(\chi_{m n} r_{i}\right)-Y_{m+1}\left(\chi_{m n} r_{i}\right)\right]\right)^{2}\right] .
\end{aligned}
$$

[1] Z.A. Daya, V. B. Deyirmenjian, S. W. Morris, and J. R. de Bruyn, Phys. Rev. Lett. 80, 964 (1998).

[2] Z. A. Daya, S. W. Morris, and J. R. de Bruyn, Phys. Rev. E 55, 2682 (1997).

[3] V. B. Deyirmenjian, Z. A. Daya, and S. W. Morris, Phys. Rev. E. 56, 1706 (1997).

[4] M. C. Cross and P. C. Hohenberg, Rev. Mod. Phys. 65, 851 (1993).

[5] D. Fulze, Adv. Geophys., 7, 1 (1961), P. Hignett, A. A. White, R. D. Carter, W. D. N. Jackson, and R. M . Small, Q. J. R. Met. Soc., 111, 131 (1985).

[6] A. Alonso, M. Net, and E. Knobloch, Phys. Fluids, 7, 935 (1995).

[7] S. Chandrasekhar, Hydrodynamic and Hydromagnetic Stability (Clarendon, Oxford, 1961).

[8] H.F. Goldstein, E. Knobloch, I. Mercader, and M. Net, J. Fluid Mech., 248, 583 (1993), 262, 293 (1994)

[9] F. Zhong, R. E. Ecke, and V. Steinberg, J. Fluid Mech., 249, 135 (1993), Phys. Rev. Lett. 67, 2473 (1991) ; R. E. Ecke, F. Zhong, and E. Knobloch, Europhys. Lett. 19, 177 (1992);

[10] E. Knobloch, in Lectures on Solar and Planetary Dynamos edited by M. R. E. Proctor and A. D. Gilbert (Cambridge University Press, New York, 1994), p. 331.

[11] S. P. Trainoff, Ph.D. thesis, University of California, Santa Barbara, 1997 (unpublished); H. W. Muller and M. Tveitereid, Phys. Rev. Lett. 74, 1582 (1995).

[12] K. Fujimura and R. E. Kelly, Fluids Dynamics Research 2, 281 (1988).

[13] S. W. Morris, J. R. de Bruyn, and A. D. May, Phys. Rev. Lett. 65, 2378 (1990).

[14] S. W. Morris, J. R. de Bruyn, and A. D. May, J. Stat. Phys. 64, 1025 (1991).

[15] S. W. Morris, J. R. de Bruyn, and A. D. May, Phys. Rev. A 44, 8146 (1991).

[16] S. S. Mao, J. R. de Bruyn, and S. W. Morris, Physica A 239, 189 (1997).
[17] S. S. Mao, J. R. de Bruyn, Z. A. Daya, and S. W. Morris, Phys. Rev. E 54, R1048 (1996).

[18] K.L. Babcock, G. Ahlers, and D.S. Cannell, Phys. Rev. E 50, 3670 (1994).

[19] N. Agrait and A. Castellanos, PhysicoChemical Hydrodynamics, 10, 181 (1988).

[20] P. G. Drazin and W. H. Reid, Hydrodynamic Stability (Cambridge University Press, Cambridge, 1989).

[21] X-l. Wu, B. Martin, H. Kellay, and W. I. Goldburg, Phys. Rev. Lett. 75, 236 (1995).

[22] A. T. Pérez and A. Castellanos, Phys. Rev. A 40, 5844 (1989), J. R. Melcher, Continuum Electromechanics, (MIT Press, Cambridge MA, 1981), N. J. Felici, J. Phys. (Paris) Colloq. 37, C1-11 (1976).

[23] Pattern Formation in Liquid Crystals, edited by A. Buka and L. Kramer (Springer, Berlin, 1995).

[24] S. Faetti, L. Fronzoni, and P. Rolla, J. Chem. Phys. 79, 5054 (1983).

[25] A. Becker, S. Ried, R. Stannarius, and H. Stegemeyer, Europhys. Lett. 39, 257 (1997).

[26] S. Ried, H. Pleiner, W. Zimmermann, and H. R. Brand, Phys. Rev. E 53, 6101 (1996).

[27] In Ref. [26], the charge density is eliminated using the Maxwell equation, so that the surface charges that we consider here are not included. The film is treated as if it were unbounded in the $z$ direction.

[28] A. P. Prudnikov, Yu. A. Brychkov, and O. I. Marichev, Integrals and Series, Vol. 2, Special Functions (Gordon and Breach, New York, 1992), p 207.

[29] I. S. Gradshetyn and I. M. Ryzhik, Integrals, Series and Products (Academic Press, New York, 1980), p 666.

[30] W. H. Press, S. A. Teukolsky, W. T. Vetterling, and B. P. Flannery, Numerical Recipes in C, (Cambridge University Press, Cambridge, 1992).

[31] P. G. de Gennes and J. Prost, The Physics of Liquid Crystals 2nd ed. (Clarendon, Oxford, 1993).

[32] To control the nature of the ionic species in the liquid crystal, octylcyanobiphenyl (8CB), was doped with tetracyanoquinodimethane (TCNQ).

[33] D. Avsec and M. Luntz, Compt. Rend. Acad. Sci. (Paris) 203, 1140 (1936).

[34] W. V. R. Malkus and G. Veronis, Phys. Fluids 4, 13 (1961).

[35] D. Dash and X.L. Wu, Phys. Rev. Lett. 79, 1483 (1997).

[36] The drift in conductivity was typically $2 \%$ over the course of a run, and is due to electrochemical reactions between the the liquid crystal and the electrodes. See S. Barret, F. Gaspard, R. Herino, and F. Mondon, J. Appl. Phys. 47, 2375 (1976).

[37] D. A. Dunmur, M. R. Manterfield, W. H. Miller and J. K. Dunleavy, Mol. Cryst. Liq. Cryst. 46, 127 (1978).

[38] We did not extrapolate our nonlocal annular calculations to the limit of an infinitely dense relaxation grid, as was done for the rectangular case in Ref. [2]. This leads to a small difference between our numerical results for $\mathcal{R}_{c}^{0}$ in the limit $\alpha \rightarrow 1$ and those for the rectangular case, for which $\mathcal{R}_{c}=91.84$.

[39] F. H. Busse, J. Fluid Mech., 44, 441 (1970). 